IIIIIII Open Access Articles

\author{
Sloshing of Galaxy Cluster Core Plasma in \\ the Presence of Self-interacting Dark Matter
}

The MIT Faculty has made this article openly available. Please share how this access benefits you. Your story matters.

\begin{tabular}{|l|l|}
\hline As Published & $10.3847 / 1538-4357 /$ AB321D \\
\hline Publisher & American Astronomical Society \\
\hline \hline Version & Final published version \\
\hline Citable link & https://hdl.handle.net/1721.1/135885 \\
\hline Terms of Use & $\begin{array}{l}\text { Article is made available in accordance with the publisher's } \\
\text { policy and may be subject to US copyright law. Please refer to the } \\
\text { publisher's site for terms of use. }\end{array}$ \\
\hline
\end{tabular}




\title{
Sloshing of Galaxy Cluster Core Plasma in the Presence of Self-interacting Dark Matter
}

\author{
J. A. ZuHone ${ }^{1}$ (1) J. Zavala ${ }^{2}$, and M. Vogelsberger ${ }^{3}$ \\ ${ }^{1}$ Harvard-Smithsonian Center for Astrophysics, 60 Garden St., Cambridge, MA 02138, USA \\ ${ }^{2}$ Center for Astrophysics and Cosmology, Science Institute, University of Iceland, Dunhagi 5, 107 Reykjavik, Iceland \\ ${ }^{3}$ Kavli Institute for Astrophysics and Space Research, Massachusetts Institute of Technology, 77 Massachusetts Ave, Cambridge, MA 02139, USA \\ Received 2019 January 30; revised 2019 July 1; accepted 2019 July 13; published 2019 September 9
}

\begin{abstract}
The "sloshing" of the cold gas in the cores of relaxed clusters of galaxies is a widespread phenomenon evidenced by the presence of spiral-shaped "cold fronts" in X-ray observations of these systems. In simulations, these flows of cold gas readily form via interactions of the cluster core with small subclusters, due to a separation of the cold gas from the dark matter (DM), due to their markedly different collisionalities. In this work, we use numerical simulations to investigate the effects of increasing the DM collisionality on sloshing cold fronts in a cool-core cluster. For clusters in isolation, the formation of a flat DM core via self-interactions results in modest adiabatic expansion and cooling of the core gas. In merger simulations, cold fronts form in the same manner as in previous simulations, but the flattened potential in the core region enables the gas to expand to larger radii in the initial stages. Upon infall, the subclusters DM mass decreases via collisions, reducing its influence on the core. Thus, the sloshing gas moves slower, inhibiting the growth of fluid instabilities relative to simulations where the DM cross section is zero. This also inhibits turbulent mixing and the increase in entropy that would otherwise result. For values of the cross section $\sigma / m \gtrsim 1$, subclusters do not survive as self-gravitating structures for more than two core passages. Additionally, separations between the peaks in the X-ray emissivity and thermal SunyaevZeldovich effect signals during sloshing may place constraints on DM self-interactions.
\end{abstract}

Key words: dark matter - galaxies: clusters: intracluster medium - methods: numerical

\section{Introduction}

A key ingredient in the standard model of cosmology is the presence of dark matter (DM), which is thought to be composed of one (or several) new particle(s) that have yet not been identified. Observations of stellar motions in galaxies, galaxy motions in clusters, and hot gas temperatures in clusters, among other astrophysical sources of evidence, imply that the total mass of these systems is nearly an order of magnitude more than is visible via electromagnetic radiation. Constraints from big bang nucleosynthesis imply that this matter must be in a non-baryonic form.

In the standard paradigm, DM is made of cold and collisionless particles ("cold dark matter," or CDM), which explains very well the large-scale structure of the universe (Springel et al. 2005, 2018; Schaye et al. 2010, 2015; Dubois et al. 2014; Vogelsberger et al. 2014a, 2014b; Davé et al. 2016; McCarthy et al. 2018). However, over the last two decades, this model has faced difficulties in describing certain aspects of cosmic structure on galactic scales, such as the the presence of low-mass galaxies with cored DM profiles, as opposed to the central cusps predicted by CDM (the "core-cusp problem"; Flores \& Primack 1994; Moore 1994; Moore et al. 1999), the wide scatter in halo profile properties (the "diversity problem"; Kuzio de Naray et al. 2010; Oman et al. 2015), and the fact that the most luminous subhalos in the Milky Way are underdense compared with CDM predictions (the "too big to fail problem"; Boylan-Kolchin et al. 2011, 2012). Explanations for these discrepancies have been put forward which involve the gravitational effects of the complex baryonic physics that impacts galaxies and their environment: stellar and supernova feedback (Mashchenko et al. 2008; Pontzen \& Governato 2012; Madau et al. 2014; Oñorbe et al. 2015; Read et al. 2016, 2019), heating from reionization (Efstathiou 1992; Bullock et al. 2000; Benson et al. 2002; Bovill \& Ricotti 2009; Sawala et al. 2016), etc. However, it is also possible that these observations are indirect evidence of new DM physics. For a recent review on the CDM challenges and a range of proposed solutions, see Bullock \& Boylan-Kolchin (2017).

One such class of CDM models that can alleviate these tensions is known as "self-interacting dark matter" (hereafter SIDM). ${ }^{4}$ In these models, the cross section for collisions between DM particles is not negligible but is large enough to produce astrophysically interesting consequences (for a recent review, see Tulin \& Yu 2018). In these models, DM particles scatter elastically and isotropically with each other, and the collisions conduct heat from the hotter, intermediate regions of the halo to the inner cold cusp. Hence, the density and velocity dispersion profiles of the central halo flatten, forming an isothermal core, at least in the early stages before triggering a runaway collapse of the core (Koda \& Shapiro 2011), the socalled gravothermal collapse in globular clusters (Lynden-Bell $\&$ Wood 1968). In order to alleviate the aforementioned tensions on galactic scales and avoid the gravothermal collapse, the self-interaction cross section per unit mass at this mass/ velocity scale should be at least $\sigma / m \sim 0.5-1.0 \mathrm{~cm}^{2} \mathrm{~g}^{-1}$, at the characteristic velocities of dwarf galaxies (Zavala et al. 2013). Since the original SIDM idea introduced by Spergel \& Steinhardt (2000) in the context of the CDM challenges, other models have been put forth that include inelastic scattering (Todoroki \& Medvedev 2019a, 2019b; Vogelsberger et al. 2019), anisotropic scattering (Robertson et al. 2017b), and velocity-dependent SIDM cross sections (Vogelsberger et al. 2012; Robertson et al. 2017b).

SIDM models are constrained more strongly in observations of massive elliptical galaxies and galaxy clusters, where

\footnotetext{
4 From here on, "CDM" will be used to refer to the standard collisionless DM model.
} 
characteristic velocities are higher, in the range 300$1000 \mathrm{~km} \mathrm{~s}^{-1}$. Self-interactions can, for instance, impact strong lensing signals $\left(\sigma / m \lesssim 0.1 \mathrm{~cm}^{2} \mathrm{~g}^{-1}\right.$, Meneghetti et al. 2001); more recently see also Despali et al. (2019), Robertson et al. (2019), and Andrade et al. (2019); evaporate massive subhalos below observed abundances $\left(\sigma / m \lesssim 0.3 \mathrm{~cm}^{2} \mathrm{~g}^{-1}\right.$, Gnedin \& Ostriker 2001); and reduce the central ellipticity of halos $(\sigma / m$ $\lesssim 1 \mathrm{~cm}^{2} \mathrm{~g}^{-1}$, Peter et al. 2013). An examination of combined stellar velocity dispersion and weak lensing measurements in the cores of clusters provided an indirect constraint of $\sigma / m \lesssim 0.1 \mathrm{~cm}^{2} \mathrm{~g}^{-1}$ (Kaplinghat et al. 2016); these observations indicate that the (allowed) radial range that can be affected by self-interactions in galaxy clusters is the inner $\mathcal{O}(10) \mathrm{kpc}$. More recently, Harvey et al. (2019) compared oscillations of brightest cluster galaxies (BCGs) in the cores of SIDM halos from cosmological simulations to observed BCG "wobbles" to derive a constraint of $\sigma / m \lesssim 0.2 \mathrm{~cm}^{2} \mathrm{~g}^{-1}$. Such observations imply that the self-interaction cross section of the DM, if non-zero, must be velocity-dependent, with higher values at smaller halo masses/ velocity scales.

Other constraints come from high-speed cluster mergers, where the relative velocity between DM particles can reach several thousand $\mathrm{km} \mathrm{s}^{-1}$. The key signatures in this case are the physical separation between baryons and the DM, optical depth arguments, and mass loss from DM interactions. As the selfinteraction cross-section increases, separations between DM and stars should increase, as the former experiences drag from collisions and the latter behaves in a collisionless fashion. The most famous example of a high-speed merging cluster is $1 \mathrm{E}$ 0657-56, or the "Bullet Cluster." Using X-ray and optical observations, a rough limit of $\sigma / m \lesssim 1 \mathrm{~cm}^{2} \mathrm{~g}^{-1}$ was suggested by Markevitch et al. (2004); by comparing $N$-body simulations with SIDM to these observations, Randall et al. (2008) was able to refine this to $\sigma / m \lesssim 1.25 \mathrm{~cm}^{2} \mathrm{~g}^{-1}$ based on the nonobservation of an offset between the DM mass peak and the galaxy centroid in the western subcluster, and $\sigma / m \lesssim$ $0.7 \mathrm{~cm}^{2} \mathrm{~g}^{-1}$ based on mass loss (see also Kahlhoefer et al. 2014). Constraints based on other merging clusters obtained using similar methods are within the range of $\sigma / m \sim 1-$ $4 \mathrm{~cm}^{2} \mathrm{~g}^{-1}$ (see Table 2 of Tulin \& Yu 2018, for a summary). Recently, refinements of the method of constraining the selfinteraction cross section in mergers have been investigated in idealized merger simulations (such as those presented in this work) by Robertson et al. (2017a) and Kim et al. (2017).

Most of the observational tests of DM collisionality on the cluster scale in terms of spatial separations of DM from baryons have focused on those locked up in stars, which should behave in a collisionless fashion. However, the collisionality of the DM can also be contrasted with that of baryons from the perspective of the X-ray-emitting hot plasma, the intracluster medium (hereafter ICM). The collisionality of the ICM in the central cluster region is much higher than that of the DM, as the latter is constrained to have a Knudsen number of $\mathcal{O}(1)$. The most famous observational example of this difference is the significant offsets between the gas and DM components in the Bullet Cluster (Markevitch et al. 2002), where the cold gas of the core of the western cluster has been pushed out of the DM core by the ram pressure of the surrounding medium, which also strips it and produces a sharp surface brightness discontinuity known as a "cold front" (for recent reviews see Markevitch \& Vikhlinin 2007; ZuHone \& Roediger 2016), where the bright/denser side of the gas is observed to be colder than the fainter/lighter side. Such cold fronts have been observed in other major merging systems.

There is another class of cluster cold fronts that also appear to depend on the different collisional properties between DM and gas. "Cool-core" clusters are relatively relaxed systems that have formed a dense, bright X-ray core, accompanied by a temperature and entropy drop down to the cluster center which have arisen from gas cooling uninterrupted by mergers. In these cores, cold fronts are often observed to be laid out in a spiral pattern if multiple fronts are observed. Simulations have shown that these fronts can be produced from interactions of the cool core with small subclusters (e.g., Ascasibar \& Markevitch 2006; ZuHone et al. 2010; Roediger et al. 2012). As a small cluster or group passes by the core, it gravitationally accelerates both the gas and DM, but these two components separate due to the ram pressure exerted on the gas by the surrounding ICM. Because the gravitational potential is dominated by the DM, after the subcluster passes the cold gas that has been uplifted from the potential minimum, it falls back toward the center, and begins an oscillatory motion which produces cold fronts. The spiral pattern of these fronts occurs due to the angular momentum transferred to the cold gas from the subcluster if (as is likely) it is not a direct head-on collision. This process and the cold fronts it produces has been dubbed "sloshing." Sloshing cold fronts have been observed in many cool-core clusters (Markevitch et al. 2000, 2003; Clarke et al. 2004; Ghizzardi et al. 2010; Simionescu et al. 2010).

The previous discussion shows that the formation of sloshing cold fronts is crucially dependent on the fact that the gas and DM have different collisionalities (Ascasibar \& Markevitch 2006). The question thus arises as to what effect an increased collisionality of the DM may have on their formation and evolution. Naively, one may suppose that making the DM more collisional would make sloshing less effective, as such collisions would effect a mild form of "ram pressure" on the DM, resulting in less of a separation between it and the gas. Thus, the presence of cold fronts in X-ray observations may potentially place a constraint on SIDM. In this work, we seek to investigate the effects of SIDM on the hot plasma of a coolcore cluster undergoing sloshing motions and producing cold fronts using hydrodynamic+DM simulations of a idealized binary cluster merger. We will show in this work that the effects of a non-zero DM cross section on the sloshing process are more complicated than the above simple picture would suggest.

The structure of this paper is as follows. In Section 2 we briefly outline the physics employed, the code details, and the setup of the galaxy cluster merger simulations. In Section 3 we present the results of our analysis, and in Section 4 we summarize these results and present our conclusions. All calculations assume a flat $\Lambda$ CDM cosmology with $h=0.71$, $\Omega_{m}=0.27$, and $\Omega_{\Lambda}=0.73$ at a redshift of $z=0$.

\section{Methods}

\subsection{Basic Physics and Code}

To perform our simulations we use the AREPO code (Springel 2010) to solve the equations of hydrodynamics and self-gravity. The former employs a finite-volume Godunov method on an unstructured moving-mesh, and the latter is computed via a Tree-PM solver. Our simulations contain three types of Lagrangian mass elements. The gas elements are 
simulated using the moving-mesh Voronoi tesselation method of AREPO. The gas is modeled as an ideal fluid with $\gamma=5 / 3$ and mean molecular weight $\mu=0.6$. Our goal in this work is to consider the effects of SIDM on the dynamics and appearance of sloshing cold fronts as seen in X-rays, so we perform our simulations in the simplest possible setting (gravity, hydrodynamics, and DM self-interactions) without the complications of additional physics such as radiative cooling and AGN feedback.

The second set of mass elements is the DM particles, which in the CDM model only interact with each other and with other matter via gravity. DM self-interactions have been incorporated into the AREPO code after the method of Vogelsberger et al. (2012). This implementation within AREPO has been used in previous works to constrain DM self-interactions at the scale of dwarf galaxies (Zavala et al. 2013; Vogelsberger et al. 2014c), to make predictions for direct DM detection experiments (Vogelsberger \& Zavala 2013), to study their effects on gravitational lensing (Díaz Rivero et al. 2018) and to study cosmological structure formation (Cyr-Racine et al. 2016; Vogelsberger et al. 2016; Lovell et al. 2018). In this work, we employ this implementation of DM self-interactions, which assumes that the scattering between DM particles is elastic and isotropic. We have chosen a constant value of the DM cross section $\sigma / m$ for all of our simulations. Such models are generally considered to be too simple, and cross sections high enough to explain observations at the galaxy scale are inconsistent with observations at the cluster scale, so velocity-dependent cross-section models are preferred (Zavala et al. 2013; Kaplinghat et al. 2016). Since our idealized simulations are focused singly on the cluster scale, a single velocity-independent value of $\sigma / m$ for each simulation is sufficient. We investigate the effects of a varying cross section by performing a number of simulations with different values of $\sigma / m=0.0,0.1,1.0,3.0$, and $10.0 \mathrm{~cm}^{2} \mathrm{~g}^{-1}$. For the $\sigma / m=$ $0 \mathrm{~cm}^{2} \mathrm{~g}^{-1}$ case we simply simulate CDM without the selfinteraction model compiled in, but we refer to it by its crosssection value as a shorthand. Given the constraints on the cross section for the clusters mentioned above, the value of $\sigma / m=$ $10 \mathrm{~cm}^{2} \mathrm{~g}^{-1}$ is definitively ruled out by observations at the cluster scale, and $\sigma / m=3 \mathrm{~cm}^{2} \mathrm{~g}^{-1}$ is nearly ruled out. These are included here as reference cases.

The third and final type of mass element we employ is star particles, which will serve as tracers of the stellar material of the cluster. In this work, we only simulate the stars associated with the BCG in the cluster center, which will serve as a useful reference frame for the dynamics of the core region and enable us to examine the relative separations of gas, stars, and DM initiated by the merger.

\subsection{Initial Conditions}

We use initial conditions for our binary cluster merger setup that are identical to those of Ascasibar \& Markevitch (2006) and ZuHone et al. (2010), which we outline here in brief.

Our merging clusters consist of a large, "main" cluster, and a small infalling subcluster. For the combined density profile $(\mathrm{DM}+$ stars $)$ of the main cluster we have chosen a Hernquist (1990) profile:

$$
\rho_{\mathrm{DM}+\mathrm{star}}(r)=\frac{M_{0}}{2 \pi a^{3}} \frac{1}{(r / a)(1+r / a)^{3}},
$$

where $M_{0}$ and $a$ are the scale mass and length of the combined $\mathrm{DM} /$ stellar halo. The Hernquist profile has the same dependence on radius in the center as the well-known NFW profile (Navarro et al. 1997), $\rho_{\mathrm{DM}} \propto r^{-1}$ as $r \rightarrow 0$, but is used here instead because it is more analytically tractable and its mass profile converges as $r \rightarrow \infty$. We also use Equation (1) for the pure-DM density profile of the subcluster.

For the stellar component of the BCG, we use an analytical approximation to a deprojected Sérsic profile given by Merritt et al. (2006):

$$
\begin{gathered}
\rho_{*}(r)=\rho_{e} \exp \left\{-d_{n_{s}}\left[\left(r / r_{e}\right)^{1 / n_{s}}-1\right]\right\}, \\
d_{n_{s}} \approx 3 n_{s}-1 / 3+0.0079 / n_{s}, \text { for } n_{s} \gtrsim 0.5,
\end{gathered}
$$

which is a good representation of the stellar mass-density profile of elliptical galaxies. We set $n_{s}=6, \rho_{e}=1.3 \times$ $10^{4} M_{\odot} \mathrm{kpc}^{-3}$, and $r_{e}=175 \mathrm{kpc}$, giving a mass $M_{*, \mathrm{BCG}} \sim$ $3 \times 10^{12} M_{\odot}$, appropriate for a $\sim 10^{15} M_{\odot}$ cluster (Merritt et al.

2006; Kravtsov et al. 2018). We ignore the stellar mass contribution from other galaxies, as we are mainly concerned with the dynamics of the different mass components in the core region. The DM density and mass profiles are then simply the difference of the combined and stellar profiles.

For the gas density, we use a phenomenological formula that can model cool-core clusters with temperature decreasing toward the cluster center (Ascasibar \& Markevitch 2006):

$$
\rho_{\mathrm{gas}}(r)=\rho_{g 0}\left(1+\frac{r}{a_{c}}\right)\left(1+\frac{r / a_{c}}{c}\right)^{\alpha}\left(1+\frac{r}{a}\right)^{\beta},
$$

with exponents

$$
\alpha \equiv-1-n \frac{c-1}{c-a / a_{c}}, \beta \equiv 1-n \frac{1-a / a_{c}}{c-a / a_{c}},
$$

where $0<c<1$ is a free parameter that characterizes the depth of the temperature drop in the cluster center and $a_{c}$ is the characteristic radius of that drop, or the "cooling radius." We set $n=5$ in order to have a constant baryon fraction at large radii, and we compute the value of $\rho_{0}$ from the constraint $M_{\mathrm{gas}} / M_{\mathrm{DM}}=\Omega_{\mathrm{gas}} / \Omega_{\mathrm{DM}}=0.12$. With this density profile and Equation (1), the corresponding gas temperature can be derived by imposing hydrostatic equilibrium.

We perform two types of simulations. In one set, we evolve the cool-core cluster in isolation for a number of different values of the self-interaction cross section to test the effect of DM collisions on the gas properties in the absence of a merger. In the second set, we perform simulations of a merger between the same cool-core cluster and a small subcluster to produce sloshing gas motions and cold fronts. In this set, we also include other simulations where the main cluster is allowed to form a DM core via self-interactions for several Gyr before undergoing the merger, and a simulation where self-interactions are not switched on until shortly before the first core passage of the two clusters. We will describe the rationale for these simulations in more detail in Section 3.2.

The two clusters are characterized by the mass ratio $R \equiv M_{1} / M_{2}=5, \quad$ where $M_{1}=M_{0} R /(1+R)$ and $M_{2}=$ $M_{0} /(1+R)$ are the masses of the main cluster and the infalling satellite, respectively. The total cluster mass $M_{0}$ is set to $1.5 \times 10^{15} M_{\odot}$. To scale the initial profiles for the two 
subclusters, the combination $M_{i} / a_{i}^{3}$ in Equation (1) is held constant. For the main cluster, we chose $a_{1}=600 \mathrm{kpc}$, $c=0.17$, and $a_{c}=60 \mathrm{kpc}$, to resemble mass, gas density, and temperature profiles typically observed in real galaxy clusters. In particular, our main cluster closely resembles A2029 (e.g., Vikhlinin et al. 2005), a hot, relatively relaxed cluster with sloshing in the cool core. The subcluster contains DM only and has a mass-density profile given by Equation (1), though in Section 3.2.5 we describe simulations where a BCG stellar component is added to it. The choice of a subcluster without a baryonic component is somewhat unusual, but we use it in this case (as in the previous works) to produce relatively undisturbed cold fronts without significant shocks or turbulence. In future papers we plan to investigate idealized merger simulations with gas-filled halos.

With the characteristics of the clusters thus defined, we may set up the particle and cell properties in the simulations. The DM and star particles all have the same mass. The gas cells are initialized to all have the same mass, though they are allowed to undergo mesh refinement and derefinement during the simulation evolution, so this condition will not remain strictly true in their case as the simulation progresses. For each of the particle/ cell positions, a random deviate $u=M(<r) / M_{\text {total }}$ is uniformly sampled in the range $[0,1]$ and the mass profile $M(<r)$ for that particular mass type is inverted to give the radius of the particle/cell from the center of the halo.

The gas cells are assigned densities and internal energies from the gas density and temperature profiles, with their initial velocities set to zero in the rest frame of the cluster. For the DM and star particles, their initial velocities are determined using the procedure outlined in Kazantzidis et al. (2004), where the energy distribution function is calculated via the Eddington formula (Eddington 1916):

$$
\mathcal{F}(\mathcal{E})=\frac{1}{\sqrt{8} \pi^{2}}\left[\int_{0}^{\mathcal{E}} \frac{d^{2} \rho}{d \Psi^{2}} \frac{d \Psi}{\sqrt{\mathcal{E}-\Psi}}+\frac{1}{\sqrt{\mathcal{E}}}\left(\frac{d \rho}{d \Psi}\right)_{\Psi=0}\right]
$$

where $\Psi=-\Phi$ is the relative potential and $\mathcal{E}=\Psi-\frac{1}{2} v^{2}$ is the relative energy of the particle. We tabulate the function $\mathcal{F}$ in intervals of $\mathcal{E}$ and interpolate to solve for the distribution function at a given energy. Given the radius of the particle, particle speeds can then be chosen from this distribution function using the acceptance/rejection method. Once particle radii and speeds are determined, positions and velocities are determined by choosing random unit vectors in $\mathfrak{R}^{3}$.

The main cluster is evolved in isolation (without selfinteractions) for several dynamical times to smooth out the initial pressure and density fluctuations of the gas particles. The resulting equilibrium profiles are essentially identical to the initial setup, and these are the initial conditions for the main cluster that we use for all of our simulations.

For the merger simulations, both objects start at a separation of $d=3 \mathrm{Mpc}$, and with an initial impact parameter $b=$ $500 \mathrm{kpc}$. The initial cluster velocities are chosen so that the total kinetic energy of the system is set to half of its potential energy, under the approximation that the objects are point masses:

$$
E \approx-\frac{1}{2} \frac{G M_{1} M_{2}}{d}=-\frac{1}{2} \frac{R}{(1+R)^{2}} \frac{G M_{0}^{2}}{d}
$$

Table 1

Simulation Parameters

\begin{tabular}{lc}
\hline \hline Total DM+Stellar Mass $\left(M_{0}\right)$ & \\
Main Cluster & $1.25 \times 10^{15} M_{\odot}$ \\
Subcluster & $2.5 \times 10^{14} M_{\odot}$ \\
Scale Radius $(a)$ & \\
Main Cluster & $600 \mathrm{kpc}$ \\
Subcluster & $350 \mathrm{kpc}$ \\
BCG Sérsic Index $\left(n_{s}\right)$ & 6 \\
BCG Scale Density $\left(\rho_{e}\right)$ & $1.3 \times 10^{4} M_{\odot} \mathrm{kpc}^{-3}$ \\
BCG Scale Radius $\left(r_{e}\right)$ & $175 \mathrm{kpc}$ \\
Cooling Radius $\left(a_{c}\right)$ & $60 \mathrm{kpc}$ \\
Temperature Drop Parameter $(c)$ & 0.17 \\
Cluster Separation $(d)$ & $3.0 \mathrm{Mpc}$ \\
Initial Impact Parameter $(b)$ & $0.5 \mathrm{Mpc}$ \\
Initial Relative Velocity $\left(v_{1}-v_{2}\right)$ & $1466 \mathrm{~km} \mathrm{~s}^{-1}$ \\
\hline
\end{tabular}

So the initial velocities in the reference frame of the center of mass are set to

$$
v_{1}=\frac{R}{1+R} \sqrt{\frac{G M_{0}}{d}} ; v_{2}=\frac{1}{1+R} \sqrt{\frac{G M_{0}}{d}} .
$$

All simulations are set within a cubical computational domain of width $L=40 \mathrm{Mpc}$ on a side, though for all practical purposes the region of interest is confined to the inner $\sim 10 \mathrm{Mpc}$.

The main cluster has $2.375 \times 10^{7}$ gas cells, each initially with mass $m_{\mathrm{gas}}=1.14 \times 10^{7} M_{\odot}$, though the mass of these cells is allowed to change slightly during the simulation evolution. The main cluster also has $8.45 \times 10^{6} \mathrm{DM}$ particles with mass $m_{\mathrm{DM}}=1.18 \times 10^{8} \mathrm{M}_{\odot}$, and $2.7 \times 10^{4}$ star particles with mass $m_{\text {star }}=1.18 \times 10^{8} M_{\odot}$. The subcluster contains $2 \times 10^{6} \mathrm{DM}$ particles of the same mass $m_{\mathrm{DM}}$. The gravitational softening length for the gas cells and the DM and stellar particles is $2 \mathrm{kpc}$.

A summary of the simulation parameters can be found in Table 1.

\section{Results}

\subsection{Single Clusters Evolved in Isolation}

In the first set of simulations, we evolve the main cluster in isolation for each of the simulated values for the DM cross section $\sigma / m$ in order to determine the effect of the selfinteractions on the profiles of the DM, stars, and gas in the absence of a merger. Figures 1-3 show spherically averaged radial profiles of the DM density, electron number density density, gas temperature, and gas entropy (defined as $S=k_{B} \operatorname{Tn}_{e}^{-2 / 3}$ ) for the three different epochs of $t=1,2$, and 5 Gyr for all of the simulated cross sections in this work. The first two epochs are significant, as they bracket the time of first core passage in the subsequent merger simulations.

The black lines in each panel show the initial profile at $t=0 \mathrm{Gyr}$. It should first be noted that for the case with $\sigma / m=0 \mathrm{~cm}^{2} \mathrm{~g}^{-1}$ (CDM, blue curves), the profiles are stable for every epoch, with the exception of the inner $\sim 10 \mathrm{kpc}$, where the gas quantities flatten out due to limited force resolution and Poisson noise. The convergence radius of the halo, outside of which discreteness effects from the force-softening and finite time step are expected to be negligible at a given epoch (Power et al. 2003), is marked by the vertical dashed line in each plot, 

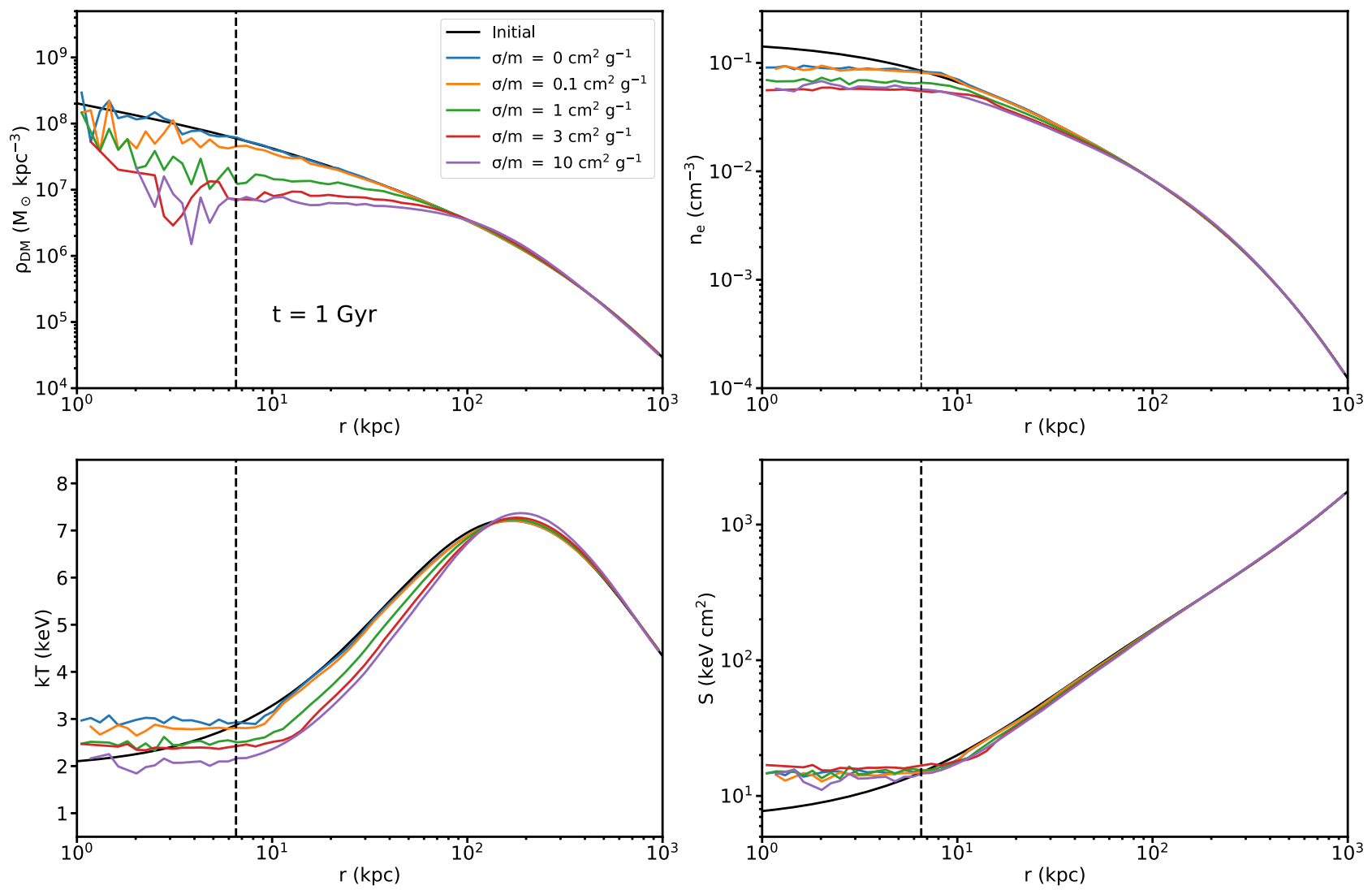

Figure 1. Spherically averaged radial profiles of various quantities at $t=1 \mathrm{Gyr}$ for the single-cluster tests for different values of $\sigma / m$ (shown with different colors as given in the legends). For each epoch, the panels show DM density, electron number density, gas temperature, and gas entropy. The solid dashed lines mark the "convergence radius" (Power et al. 2003) at each epoch.

and is $\sim 6.5 \mathrm{kpc}$ or less (depending on the epoch), implying that our radial profiles are converged roughly outside this radius. This is also implied by the stability of the profiles for the collisionless simulation. Non-zero values of the DM crosssection result in a flattening of the DM core density, which happens more quickly for larger values of $\sigma / m$ but the size of the core at later times tends toward the same for all cross sections (as in Robertson et al. 2017a). At very late times, for the largest cross sections the DM density slowly begins to increase again due to the "gravothermal catastrophe" inherent in any selfgravitating system where collisions can carry energy away from the core region of the system i.e., with negative heat capacity (Lynden-Bell \& Wood 1968; Kochanek \& White 2000).

Since the flattening of the DM core is a gradual process, the response of the gas to the changing gravitational potential is an adiabatic expansion - the gas density in the core decreases and the temperature decreases (outside of the inner $\sim 10 \mathrm{kpc}$ as noted above). These effects are more pronounced for larger SIDM cross sections. However, the changes in the gas density and temperature are rather small, roughly a factor of $\sim 2$ at most, so the system retains its identity as a "cool-core" cluster. The gas entropy profile is essentially the same across the simulations, consistent with the assumption that the changes are adiabatic. At larger radii near $r \sim 200 \mathrm{kpc}$, where the DM density increases beyond its initial value, an adiabatic compression of the gas occurs, and the temperature and density in this region increase. This effect is most pronounced for the $\sigma / m=10 \mathrm{~cm}^{2} \mathrm{~g}^{-1}$ simulation.

These results point to an important factor affecting the evolution of our idealized merger simulations, which we will describe in the next section-even before the first core passage between the main cluster and the subcluster, DM, gas, and stars are already evolving in ways that may affect our conclusions. Specifically, for a significant DM cross section, the sloshing stage will begin when the main cluster already has a DM core and a slightly colder, more diffuse gas core. It will be important to remember the effects of this evolution in the next section where we examine the merger simulations.

\subsection{Merger Simulations}

\subsubsection{Visible Appearance of the Cold Fronts and the Sloshing Motions}

We will first describe the visual appearance of the sloshing motions and the cold fronts they produce in the different simulations. Figures 4 and 5 show slices of gas temperature for four different epochs of the simulations for different values of the DM cross section. Contours of DM density are overlaid on these slices, which are centered on the center of mass of the BCG and focus on the core region. The bottom right panel of each epoch set in these figures also shows the ratio of the mass of gas at a given entropy at that epoch to that at the same entropy at $t=0$ for the different simulations. Since all of the simulations start with the same initial condition, this allows us to track how the different DM cross sections affect the evolution of the gas entropy.

At $t=1.5 \mathrm{Gyr}$, shown at the top of Figure 4 , the subcluster has recently made its closest approach to the main cluster center (at $t \approx$ $1.35 \mathrm{Gyr}$ ). It has compressed and heated gas behind it in a "sonic wake" (an effect first noted by Ascasibar \& Markevitch 2006), 

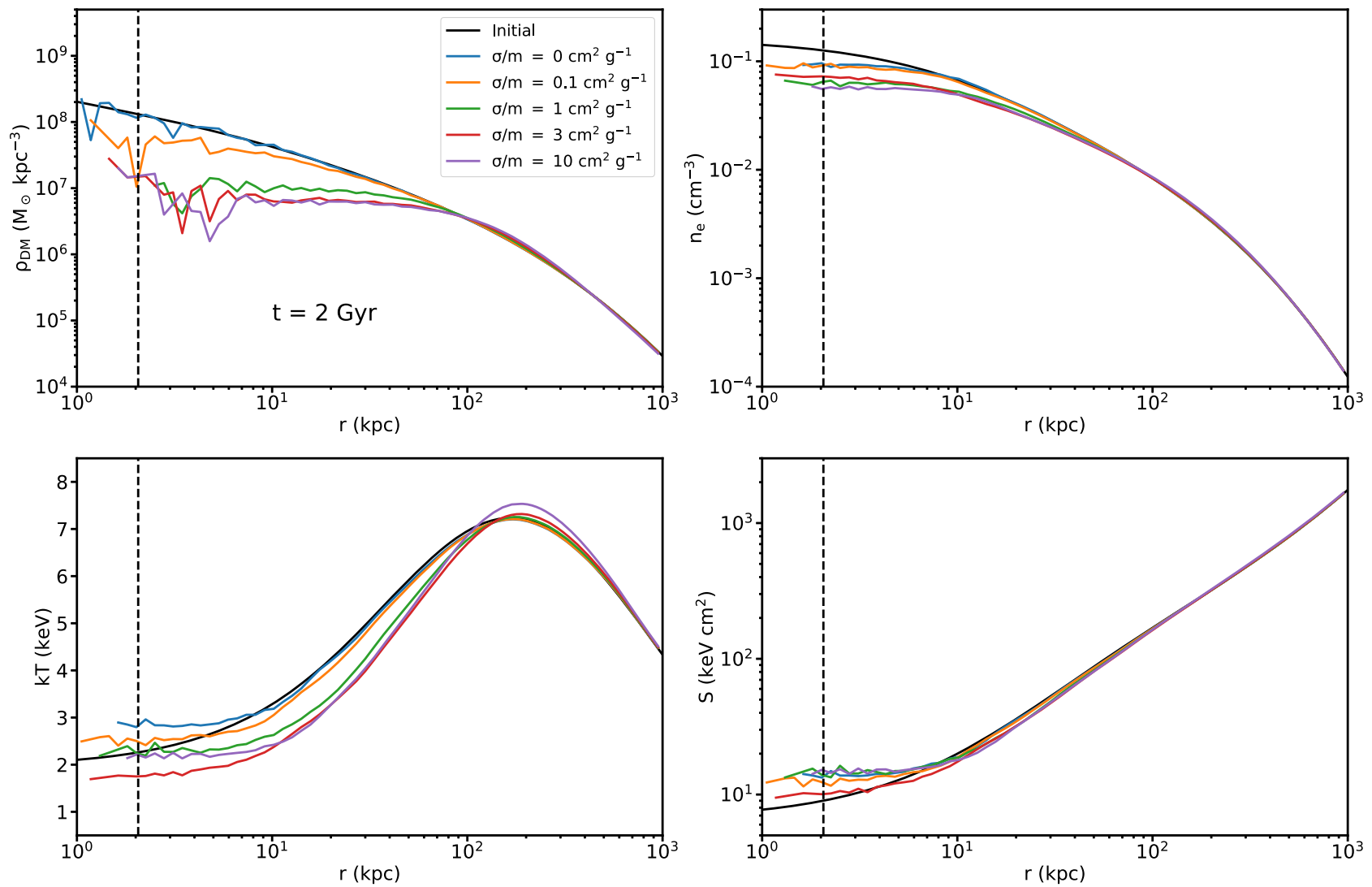

Figure 2. Spherically averaged radial profiles of various quantities at $t=2 \mathrm{Gyr}$ for the single-cluster tests for different values of $\sigma / m$ (shown with different colors as given in the legends). For each epoch, the panels show DM density, electron number density, gas temperature, and gas entropy. The solid dashed lines mark the "convergence radius" (Power et al. 2003) at each epoch.
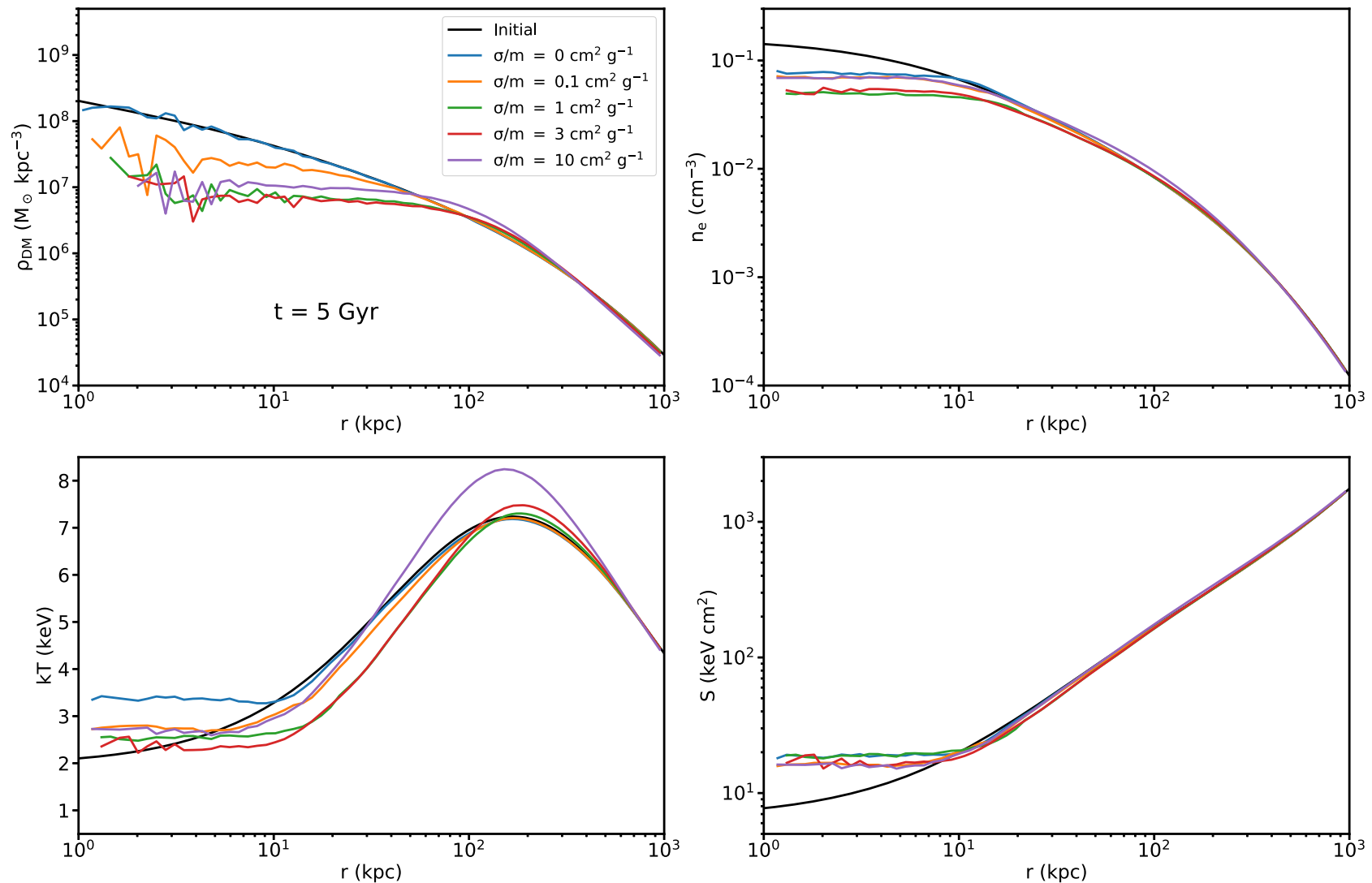

Figure 3. Spherically averaged radial profiles of various quantities at $t=5 \mathrm{Gyr}$ for the single-cluster tests for different values of $\sigma / m$ (shown with different colors as given in the legends). For each epoch, the panels show DM density, electron number density, gas temperature, and gas entropy. 

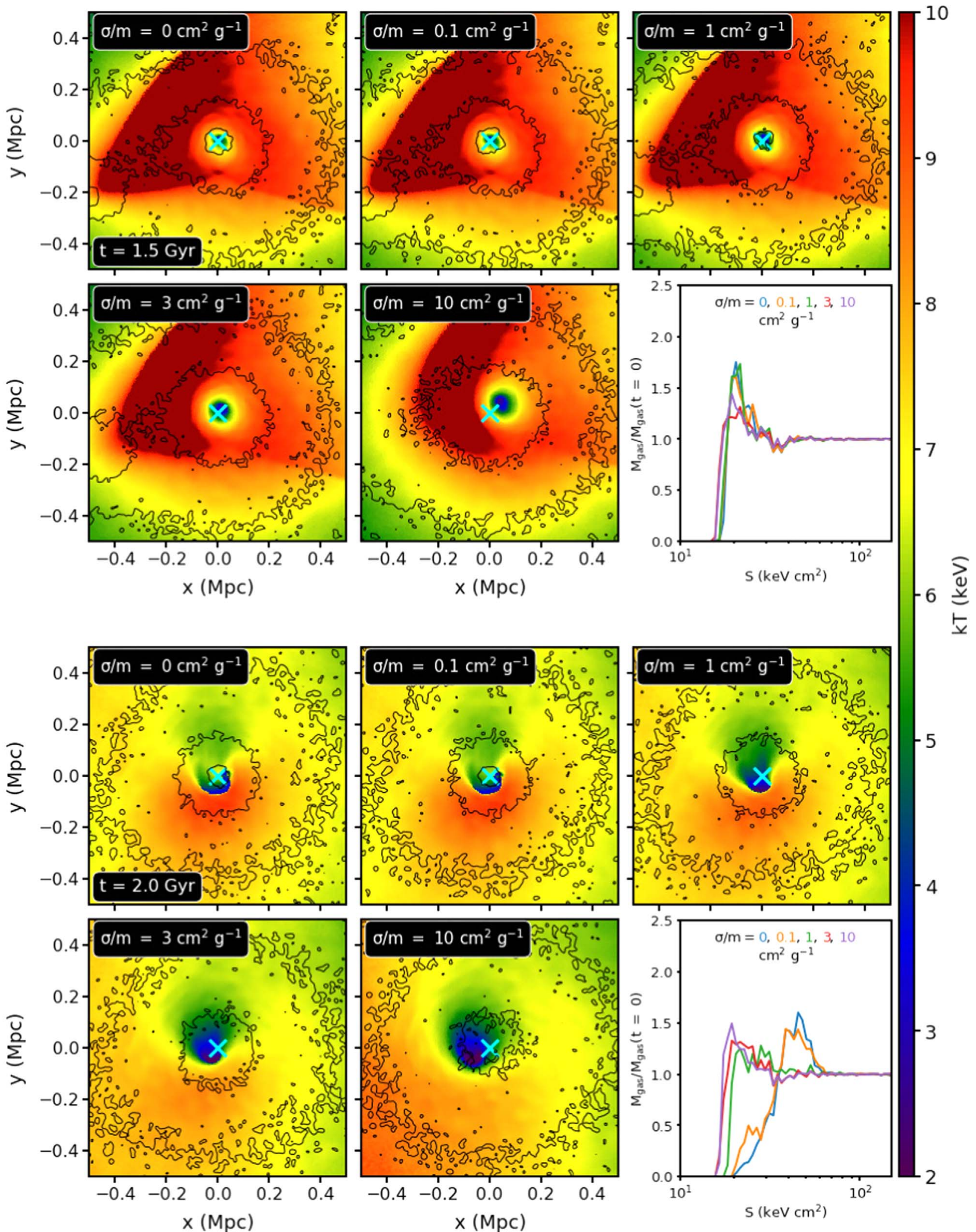

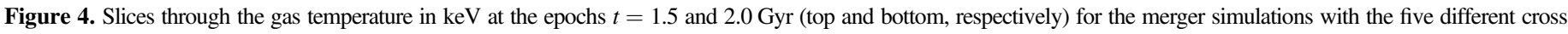

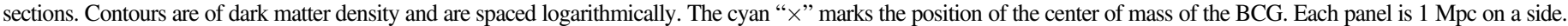

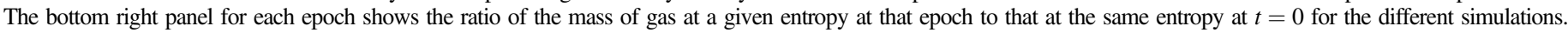

which has a fairly similar appearance in all five simulations. This sonic wake is responsible for transferring angular momentum to the cold gas in the core. For larger values of $\sigma / m$, the subcluster has already become far less centrally concentrated due to collisions, which are particularly enhanced during the core passage. The trajectory of the subcluster is only moderately altered by 

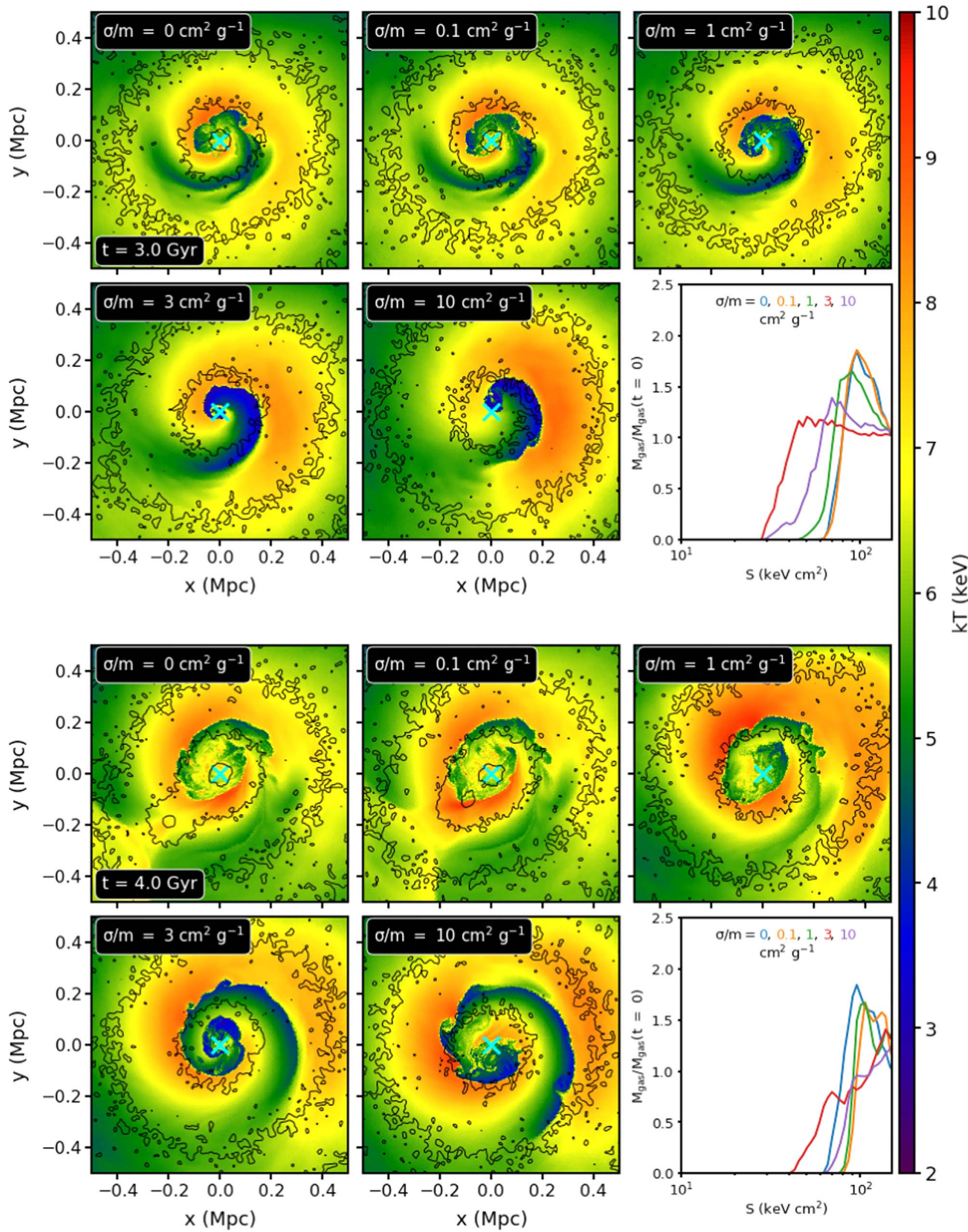

Figure 5. Slices through the gas temperature in $\mathrm{keV}$ at the epochs $t=3.0$ and $4.0 \mathrm{Gyr}$ (top and bottom respectively) for the merger simulations with the five different cross sections. Contours are of dark matter density and are spaced logarithmically. The cyan " $\times$ " marks the position of the center of mass of the BCG. Each panel is 1 Mpc on a side. The bottom right panel for each epoch shows the ratio of the mass of gas at a given entropy at that epoch to that at the same entropy at $t=0$ for the different simulations.

self-interactions at this point, with the exception of the $\sigma / m=$ $10 \mathrm{~cm}^{2} \mathrm{~g}^{-1}$ case, where it has been slowed down significantly by the drag force to due to the large number of collisions at core passage. For the largest values of $\sigma / m=3$ and $10 \mathrm{~cm}^{2} \mathrm{~g}^{-1}$, the wake has become detached from the subcluster at this stage. We also note that in the case of $\sigma / m=10 \mathrm{~cm}^{2} \mathrm{~g}^{-1}$ the cool core has 

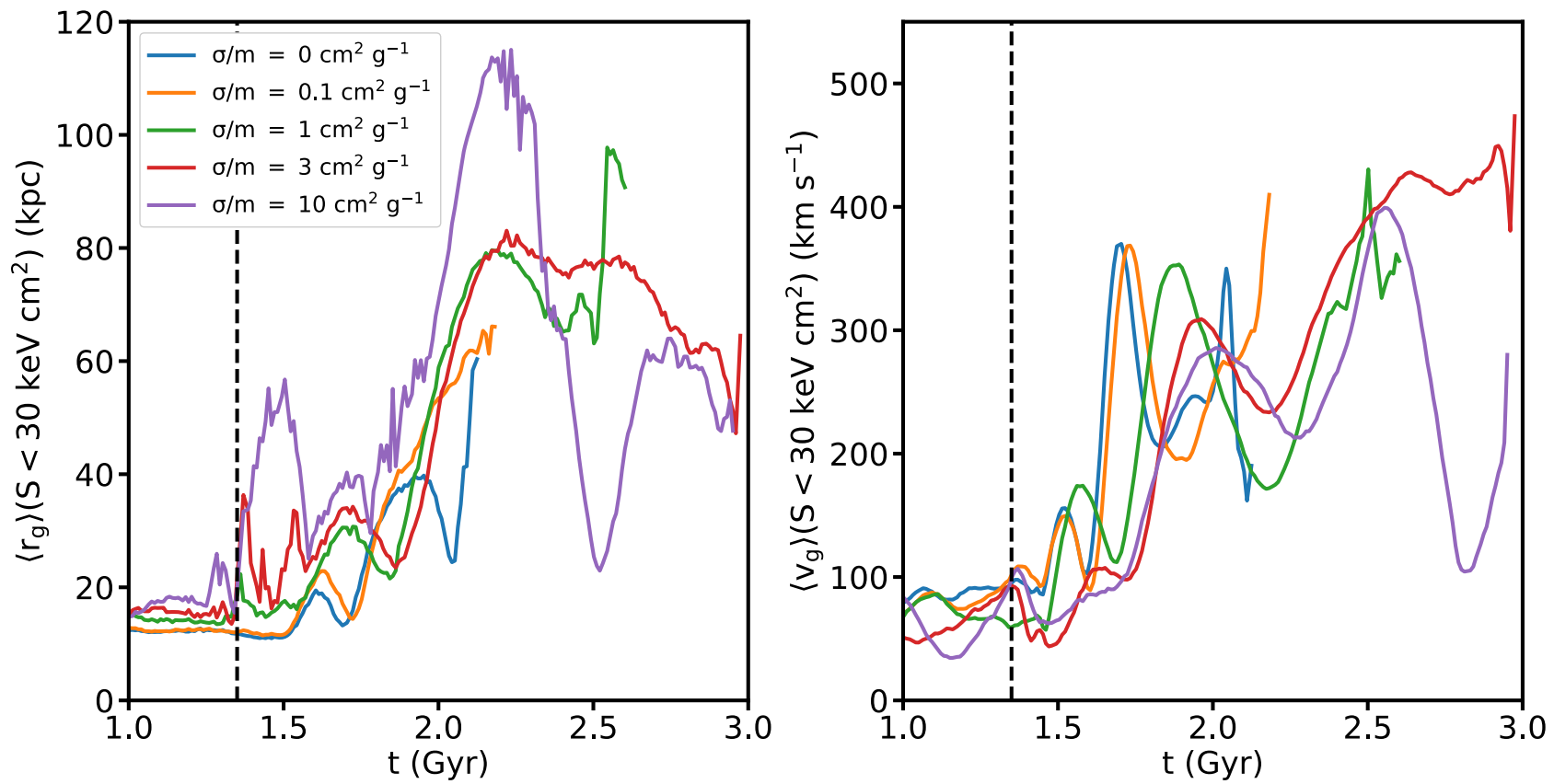

Figure 6. Evolution of the mass-weighted average radius and velocity (with respect to the center of mass frame of the BCG) of gas with $S \leqslant 30 \mathrm{keV} \mathrm{cm}^{2}$ for the simulations with different $\sigma / m$. The vertical dashed line marks the approximate epoch of core passage at $t=1.35 \mathrm{Gyr}$.

already been pushed away from the BCG center by as much as roughly $50 \mathrm{kpc}$. At this early epoch, the mass distribution of entropy between the simulations is still very similar.

At $t=2 \mathrm{Gyr}$ (at the bottom in Figure 4), the process of sloshing has begun in earnest. The evolution of the cold fronts proceeds faster for lower values of the cross section, as evidenced by the presence of a sharper temperature gradient in the images between the cold (blue) and hot (orange) gas in these simulations. In general, for larger cross sections, the temperature of the lowest-entropy gas is colder by about $\sim 1 \mathrm{keV}$, consistent with the result from Section 3.1 which showed that the slow transition from DM cusp to core resulted in adiabatic expansion and cooling of the most central gas. This lower temperature in the SIDM cases is somewhat artificial and is a consequence of our idealized setup-in Section 3.2.5 we will investigate how much our conclusions depend on it.

The two simulations with $\sigma / m=0,0.1 \mathrm{~cm}^{2} \mathrm{~g}^{-1}$ have already lost a substantial mass of gas with $S \lesssim 30 \mathrm{keV} \mathrm{cm}^{2}$, presumably due to mixing of hot and cold gas, whereas the other simulations have retained this low-entropy gas. Consistent with this, these two simulations are already showing early signs of the Kelvin-Helmholtz instability (hereafter KHI), as seen in the slice images for those cross sections.

At $t=3 \mathrm{Gyr}$ (shown at the top of Figure 5), the cold fronts are very well-developed in all of the simulations. The spatial extent of the fronts is very nearly the same in all simulations, indicating they travel outward with roughly the same radial velocity. Important differences are present, however. First, the cold fronts in the simulations with larger $\sigma / m$ are still noticeably colder by about $\sim 1 \mathrm{keV}$. Second, at this epoch, it is more obvious that the simulations with larger cross sections are less susceptible to the KHI and correspondingly appear smoother than those with lower cross sections. These enhanced $\mathrm{KHI}$ in the less collisional simulations result in "box-shaped" cold fronts and enhanced turbulence and gas mixing, as noted in previous works (e.g., ZuHone et al. 2010; Roediger et al. 2012). We will discuss the reason for this somewhat surprising dependence of the KHI on the DM cross section in Section 3.2.2. The effect of this reduced mixing is shown in the distribution of entropy at this epoch, as the simulations with $\sigma / m \leqslant 1 \mathrm{~cm}^{2} \mathrm{~g}^{-1}$ have less low-entropy gas than the higher cross sections, as shown in the bottom right panel of the top part of Figure 5). However, the trend is somewhat reversed in the extreme case of $\sigma / m=10 \mathrm{~cm}^{2} \mathrm{~g}^{-1}$, as it has lost more lowentropy gas than the $\sigma / m=3 \mathrm{~cm}^{2} \mathrm{~g}^{-1}$ case, and also appears slightly more susceptible to KHI. It should be noted that in this simulation the flattening of the potential is most extreme, and the modest stabilizing effect against KHI provided by the gravitational force is greatly reduced here.

At later times, $t \gtrsim 4$ Gyr (shown at the bottom of Figure 5), the outermost cold fronts have traveled out to a radius where the density profile of the DM is essentially identical across the simulations with varying $\sigma / m$, thus the subsequent evolution is similar in appearance. The colder gas in the simulations with larger cross sections has persisted even to this later time. At this epoch, in all simulations KHI rolls appear at the cold front surfaces. It is also around this epoch, in the simulations with $\sigma / m \leqslant 1 \mathrm{~cm}^{2} \mathrm{~g}^{-1}$, that the subcluster makes a second core passage, moderately heating the core once more and driving a shock front. In the $\sigma / m=3$ and $10 \mathrm{~cm}^{2} \mathrm{~g}^{-1}$ simulations, the subcluster DM has been completely evaporated into the surrounding main cluster DM via collisions by this time, which will be discussed in more detail in Section 3.2.4. By this time, all simulations have lost significant amounts of lowentropy gas due to KHI and turbulent mixing.

\subsubsection{Phase Space Trajectories of the Cold Gas}

From the results above, the behavior of the coldest gas in the core is clearly strongly dependent on the effect that selfinteractions have on the gravitational potential in the core. Figure 6 shows the evolution of the average radius and velocity of the lowest-entropy gas in the cluster core (the averages are taken over all gas with $S \leqslant 30 \mathrm{keV} \mathrm{cm}^{2}$ ) with respect to the center of mass frame of the BCG. Both quantities increase with 


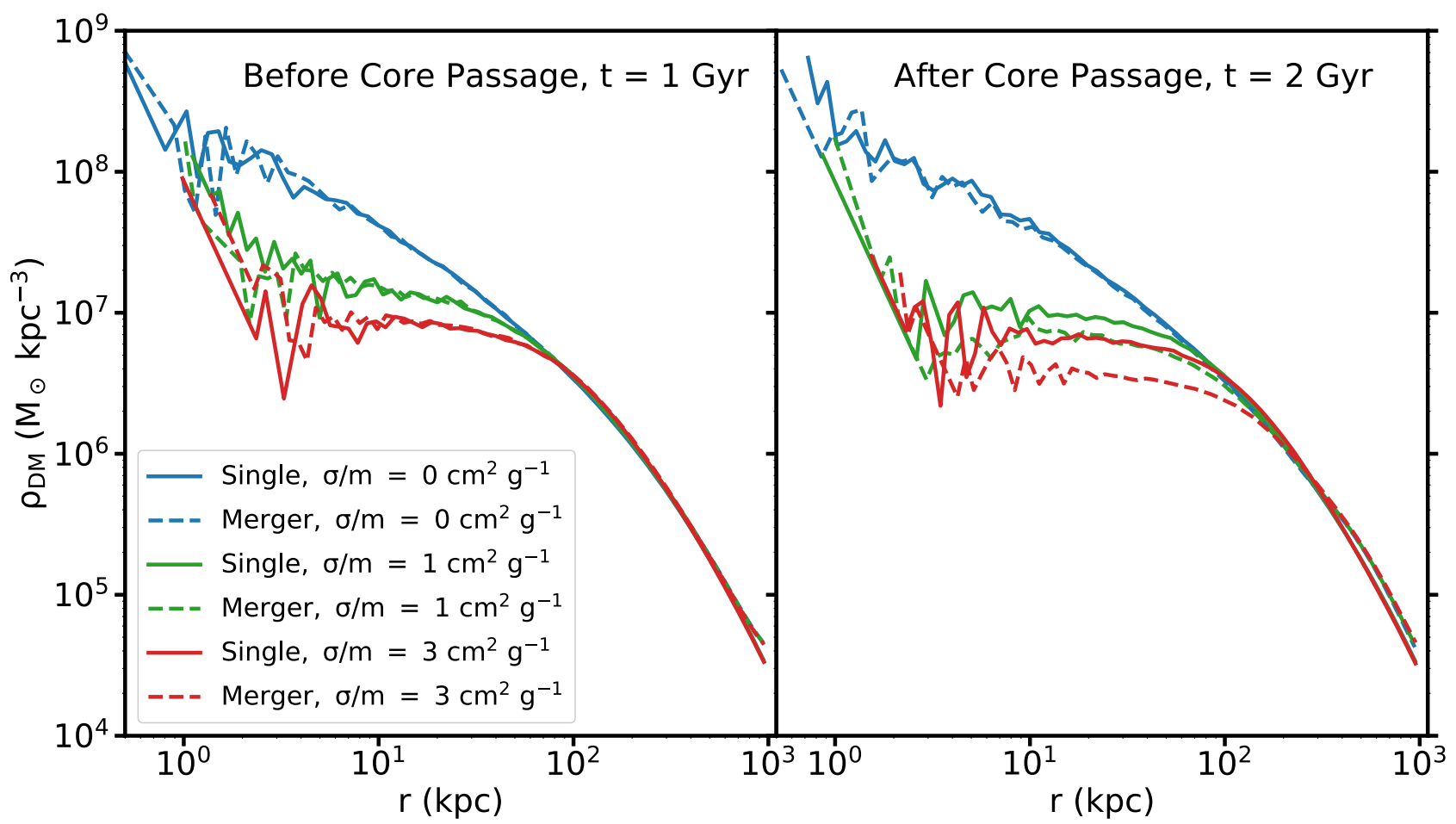

Figure 7. Spherically averaged profiles of the DM density centered on the main cluster potential minimum before and after core passage for the single-cluster and merger runs for three different values of the DM cross section.

time, though superimposed on this increase is an oscillatory motion as the gas sloshes back and forth in the potential. It should be noted that it is not the case that the same low-entropy gas is gradually rising with radius, which would violate the Schwarzschild stability condition $d S / d r>0$. Instead, the average entropy of this gas within $S \leqslant 30 \mathrm{keV} \mathrm{cm}^{2}$ is increasing within this limit as cold, low-entropy gas mixes with hot, high-entropy gas from larger radii and the entropy of the core as a whole gradually rises.

The time period from the core passage at $t \approx 1.35 \mathrm{Gyr}$ up to $t \sim$ $2 \mathrm{Gyr}$ is crucial for the development of the cold fronts. In general, for larger values of the DM cross section, the coldest gas is able to rise to larger radii during this period. This difference in radial extent is significant-by $t \sim 2 \mathrm{Gyr}$, the lowest-entropy gas has risen to only $\sim 40 \mathrm{kpc}$ in the $\sigma / m=0 \mathrm{~cm}^{2} \mathrm{~g}^{-1}$ simulation, but in the $\sigma / m=1$ and $3 \mathrm{~cm}^{2} \mathrm{~g}^{-1}$ simulations it has risen to $\sim 60 \mathrm{kpc}$, and in the $\sigma / m=10 \mathrm{~cm}^{2} \mathrm{~g}^{-1}$ simulation it has risen to $\sim 70 \mathrm{kpc}$. Increasing the DM cross section has the opposite effect on the speed of the cold gas with respect to the BCG rest frame. This speed tends to be slower during this period as $\sigma / m$ increases. This effect is similarly dramatic - at $t \sim 1.75 \mathrm{Gyr}$ the speed of the cold gas in the $\sigma / m=0$ and $0.1 \mathrm{~cm}^{2} \mathrm{~g}^{-1}$ simulations is $\sim 400 \mathrm{~km} \mathrm{~s}^{-1}$, but in the higher cross-section simulations the speed is $\sim 100 \mathrm{~km} \mathrm{~s}^{-1}$

The explanation for these apparently contradictory behaviors is subtle but straightforward. The flattening of the DM density in the core region, which occurs due to DM self-interactions, leads to a flattening of the gravitational potential. It is therefore easier for the ram pressure of the surrounding medium to push the gas core out of the DM core toward larger radii against the decreased gravitational force.

The decrease in core gas speed with increasing cross section is due to the fact that as the subcluster falls into the main cluster, its mass is further reduced by frequent high-velocity collisions with particles from the main cluster's DM during its infall. These interactions are most significant during the short interval of time near the core passage (where the ambient density, and thus the scattering rate, is higher). Thus, the core gas of the main cluster experiences a reduced gravitational acceleration from the passing subcluster, and the sonic wake that is formed by the subcluster and transfers angular momentum to the cold gas is weakened. The fact that this gas is pushed to a larger radius has little effect on its velocity, as the gravitational potential gradient in this region is considerably reduced due to the flattening of the core. This effect is illustrated in more detail in Section 3.2.5.

Though this gas is moving slower, it nevertheless reaches larger radii than those in the simulations with a lower DM cross section, because in the latter simulations in the same time frame it has already reached its peak radius and fallen back into the center. The slower increase of velocity of the cold gas for large cross sections explains why in these simulations KHI appears to be suppressed until later times - the decreased velocity shear across the cold front surface results in an increased growth time for the development of KHI. In general, the slower motions and inhibited KHI associated with the larger cross sections result in less turbulent mixing of the cold gas with hotter gas, as is seen in Figure 6 by the fact that the lower-entropy gas persists longer in these simulations.

We note that the minor merger we have simulated is not sufficient to result in measurable offsets between the DM core and the BCG-any observable separation would require a direct hit of the subcluster instead of a large impact parameter and possibly a smaller mass ratio (more equal masses) between the two components (see Kim et al. 2017, for a detailed analysis of the required conditions for such separations). In a future paper we will examine such separations between gas, $\mathrm{DM}$, and stars in major mergers. 

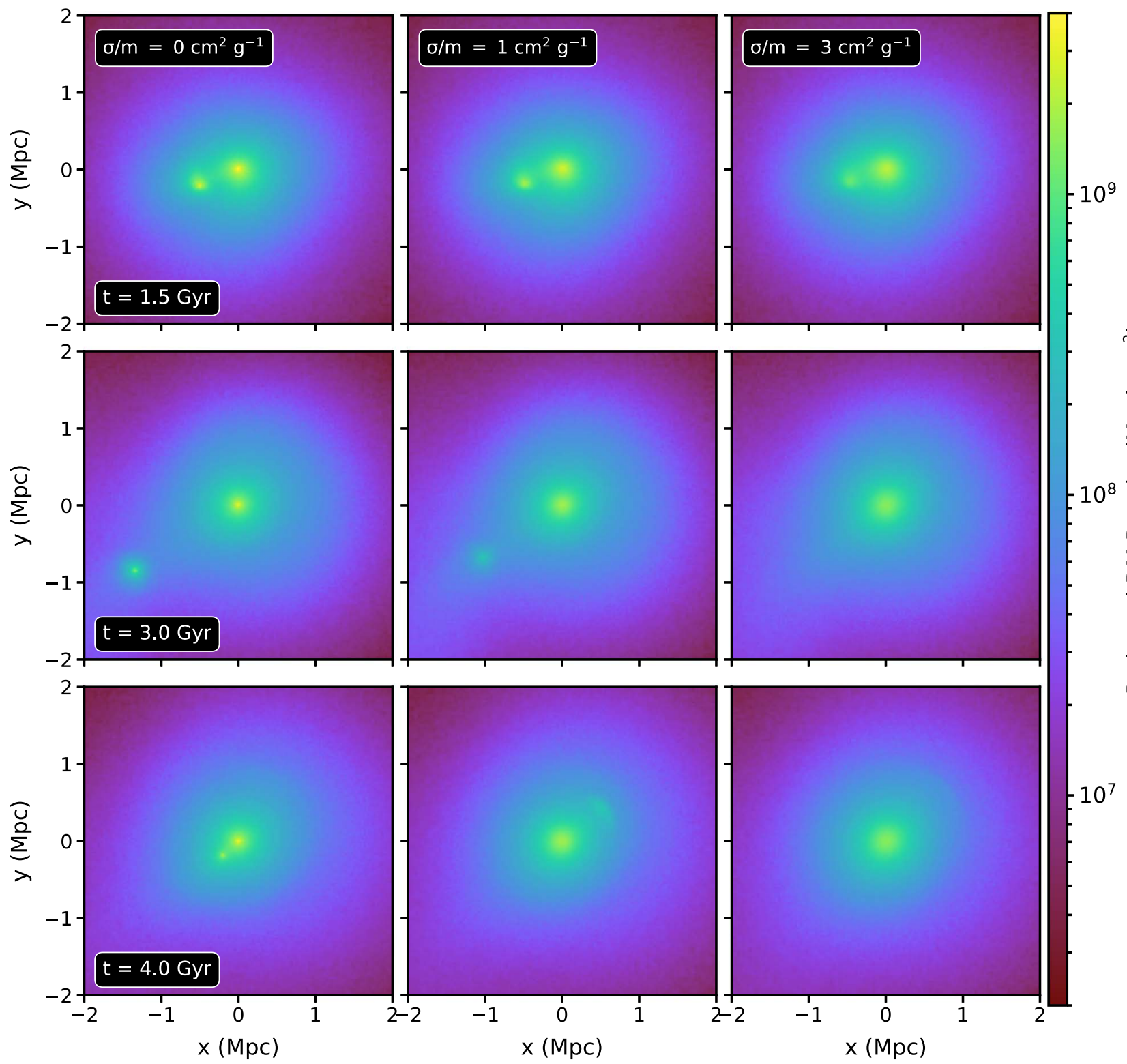

Figure 8. Projected DM density at the epochs $t=1.5,3.0$, and $4.0 \mathrm{Gyr}$ for three different values of $\sigma / \mathrm{m}$. Each panel is $4 \mathrm{Mpc}$ on a side.

\subsubsection{Effect of the Core Passage on the Main Cluster DM Core}

In our simulations, the main cluster's DM core undergoes a transformation from a higher-density cuspy profile to a lowerdensity cored profile due to DM self-interactions, whether during a merger or in isolation. During the first core passage, the DM particles from the main cluster core come into contact with those from the subcluster at high relative speed. The high density of the subcluster and the high relative speed of the collisions both increase the number of collisions and the likelihood that these collisions can eject DM particles from the core, further reducing the core density. Figure 7 shows this effect. The left panel shows the spherically averaged DM density profile for the single-cluster and merger simulations for three different values of the DM cross section, at $t=1 \mathrm{Gyr}$, shortly before the first core passage of the subcluster. The density profiles are the same between the single-cluster and merger simulations at this epoch, as expected. The right panel shows the same profiles after the core passage, at $t=2 \mathrm{Gyr}$.
When there are no collisions $\left(\sigma / m=0 \mathrm{~cm}^{2} \mathrm{~g}^{-1}\right)$, the density profile is unchanged. For $\sigma / m=1$ and $3 \mathrm{~cm}^{2} \mathrm{~g}^{-1}$, the increased number of collisions has in fact decreased the density of the core, but only slightly, by roughly a factor of $\sim 1.5-2$ at most. It should be noted that this minor change is consistent with an encounter with a subcluster 5 times less massive at a relatively high initial impact parameter of $0.5 \mathrm{Mpc}$. Mergers with more equal masses and smaller impact parameters will result in stronger effects on the DM core after the first core passage-exploration of these scenarios will be the subject of a follow-up paper.

\subsubsection{Disappearance of the Subcluster Due to DM Self-interactions}

As mentioned above, the subcluster makes a second core passage at $t \approx 3.6-4.1 \mathrm{Gyr}$. In the simulations with $\sigma / m \leqslant$ $1 \mathrm{~cm}^{2} \mathrm{~g}^{-1}$, the subcluster survives as a more or less coherent structure. In the simulations with $\sigma / m=3$ and $10 \mathrm{~cm}^{2} \mathrm{~g}^{-1}$, collisions are so frequent that the subcluster loses its coherent 
structure shortly after the core passage and becomes a stream of particles within the main cluster's DM. Even in the $\sigma / m=1 \mathrm{~cm}^{2} \mathrm{~g}^{-1}$ case, the subcluster is undergoing a complete disruption following the second core passage. The evolution of the subcluster for simulations with different values of the DM cross section is illustrated in Figure 8, which shows the projected DM density at three epochs following the core passage.

If there is no second core passage, then there will not be a second perturbation of the subcluster on the main cluster core. Since in our simulations the subcluster is gas-free, this second passage has a minor effect on the subsequent evolution of the cold fronts. More intriguingly, it is of note that in most observational accounts of sloshing cold fronts, identifying the subcluster that produced the original perturbation (typically via finding a second, smaller X-ray peak or a clump of galaxies) is often difficult. Examples include Virgo (Roediger et al. 2011) and A2204 (Chen et al. 2017), though A1644 (Johnson et al. 2010) is a notable exception which has an obvious subcluster candidate, though the stage of the sloshing motions appears very early in this case. Our results show that for non-negligible but observationally permitted values of the DM cross section $\left(\sigma / m \lesssim 1 \mathrm{~cm}^{2} \mathrm{~g}^{-1}\right)$ small subclusters (and their associated gas and galaxies) may become somewhat subsumed into the main cluster after the second core passage, providing a partial explanation for the difficulty of identifying them in observations. However, a systematic study of the optical components of clusters with sloshing cold fronts is required before any conclusions can be definitively made.

\subsubsection{Testing Alternative Scenarios}

In the last two sections, it was determined that the main effects of SIDM on sloshing motions and cold fronts in a cluster core are the flattening of the potential well of the main cluster and the stripping of mass of the subcluster during infall. Because the cluster DM cores are already softening due to selfinteractions from the very beginning of the simulation, the conclusions we draw from our merger simulations may depend in a crucial way on this evolution. In this section, we will describe the results of several other simulations we have run to test the robustness of our conclusions to variations in the premerger evolution.

The simplest alternative simulation is one where the main cluster halo is not initially cusp-shaped in the core region but already has a density profile that flattens out toward the center. For this simulation we have taken the single-cluster simulation with $\sigma / m=3 \mathrm{~cm}^{2} \mathrm{~g}^{-1}$ and taken its state at $t=5 \mathrm{Gyr}$ to be the state of the main cluster at the beginning of the merger simulation. Importantly, we also run the subcluster in isolation for several Gyr so that it too develops a flat DM core. We have run two versions of this simulation, one without any selfinteractions for the entire simulation and another where $\sigma / m=3 \mathrm{~cm}^{2} \mathrm{~g}^{-1}$. In theory, this simulation should be very similar to the $\sigma / m=3 \mathrm{~cm}^{2} \mathrm{~g}^{-1}$ case, as in that simulation the cored halo has already established itself before core passage. Figure 9 shows the evolution of the temperature of the lowestentropy gas for these simulations (dashed lines) compared to the default versions that begin with cuspy profiles (solid lines). Just before core passage, the temperatures of the lowestentropy gas are identical in the cored simulations to the original $\sigma / m=3 \mathrm{~cm}^{2} \mathrm{~g}^{-1}$ simulation, and the subsequent evolution of this temperature is very similar.

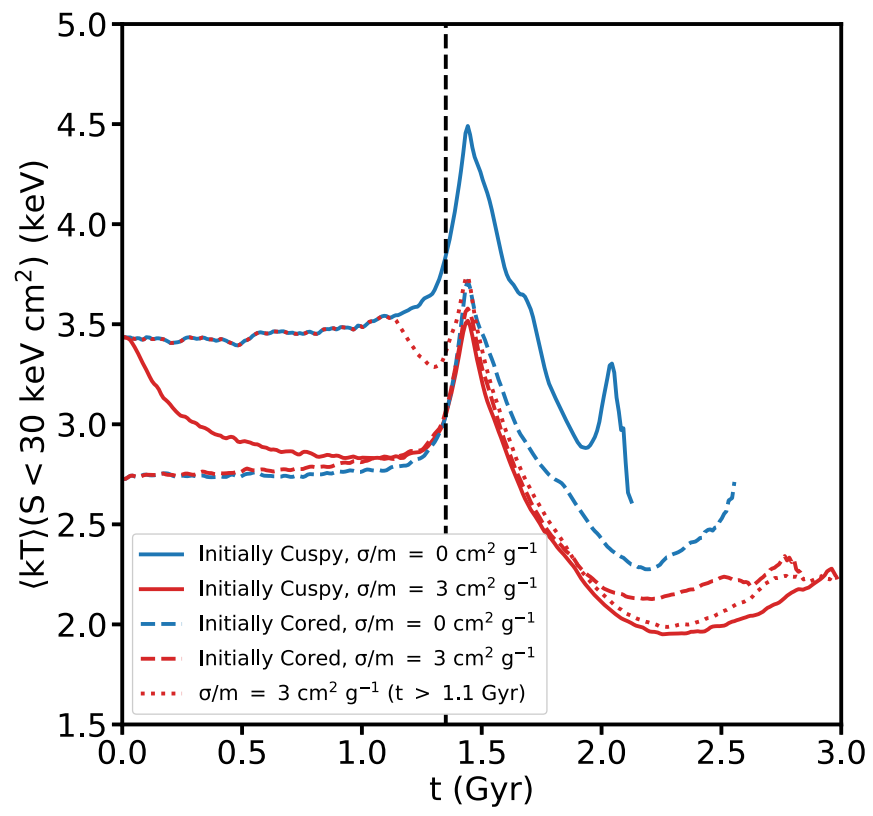

Figure 9. Evolution of the mass-weighted average temperature of gas with $S \leqslant 30 \mathrm{keV} \mathrm{cm}^{2}$ for the simulations that test alternative scenarios. The vertical dashed line marks the approximate epoch of core passage at $t=1.35 \mathrm{Gyr}$.

However, the subsequent appearance of the cold fronts themselves nevertheless still depends on whether or not the $\mathrm{DM}$ is undergoing self-interactions, regardless of the shape of the inner DM density profile. Figure 10 shows the appearance of the cold fronts at $t=3 \mathrm{Gyr}$ for the DM cusp and DM core simulations with different cross sections. In both simulations without self-interactions, the cold fronts appear very similarboth have been disturbed by KHI. In both simulations with $\sigma / m=3 \mathrm{~cm}^{2} \mathrm{~g}^{-1}$, the cold fronts appear much smoother. Though the temperature of the lowest-entropy gas is similar between the two simulations with initially flat DM profiles (from Figure 9), the overall temperature of the core is hotter in the simulation with $\sigma / m=0 \mathrm{~cm}^{2} \mathrm{~g}^{-1}$ due to the enhanced KHI driving small-scale turbulence and mixing of hot and cold gas phases. The lower right panel in Figure 10 confirms this by showing that the loss of low-entropy gas due to turbulent mixing is driven essentially exclusively by the presence of selfinteractions and not the shape of the core potential.

We also performed another simulation where the DM selfinteractions (with $\sigma / m=3 \mathrm{~cm}^{2} \mathrm{~g}^{-1}$ ) were not switched on until $t=1.1 \mathrm{Gyr}$, which is right before the core passage at $t \approx 1.35$ Gyr. Though this is a very artificial setup, it avoids the evolution of the gas and DM properties that occur due to selfinteractions within the main cluster alone during the period of the subcluster's initial approach. The dotted red line in Figure 9 shows the temperature of the lowest-entropy gas in this simulation, which begins to adiabatically cool right after the self-interactions are switched on at $t=1.1 \mathrm{Gyr}$. Its subsequent evolution is nearly identical to the other two simulations with self-interactions, and the appearance of the cold fronts at later times is also very similar to that in these simulations, as seen in Figure 10.

These results point to the fact that during a merger, selfinteractions are a critical effect beyond simply creating DM cores. This is illustrated clearly in Figure 11, which shows the projected DM density in the core region shortly after core passage for the initially cuspy simulation with $\sigma / m=3 \mathrm{~cm}^{2} \mathrm{~g}^{-1}$ (left panel), and 

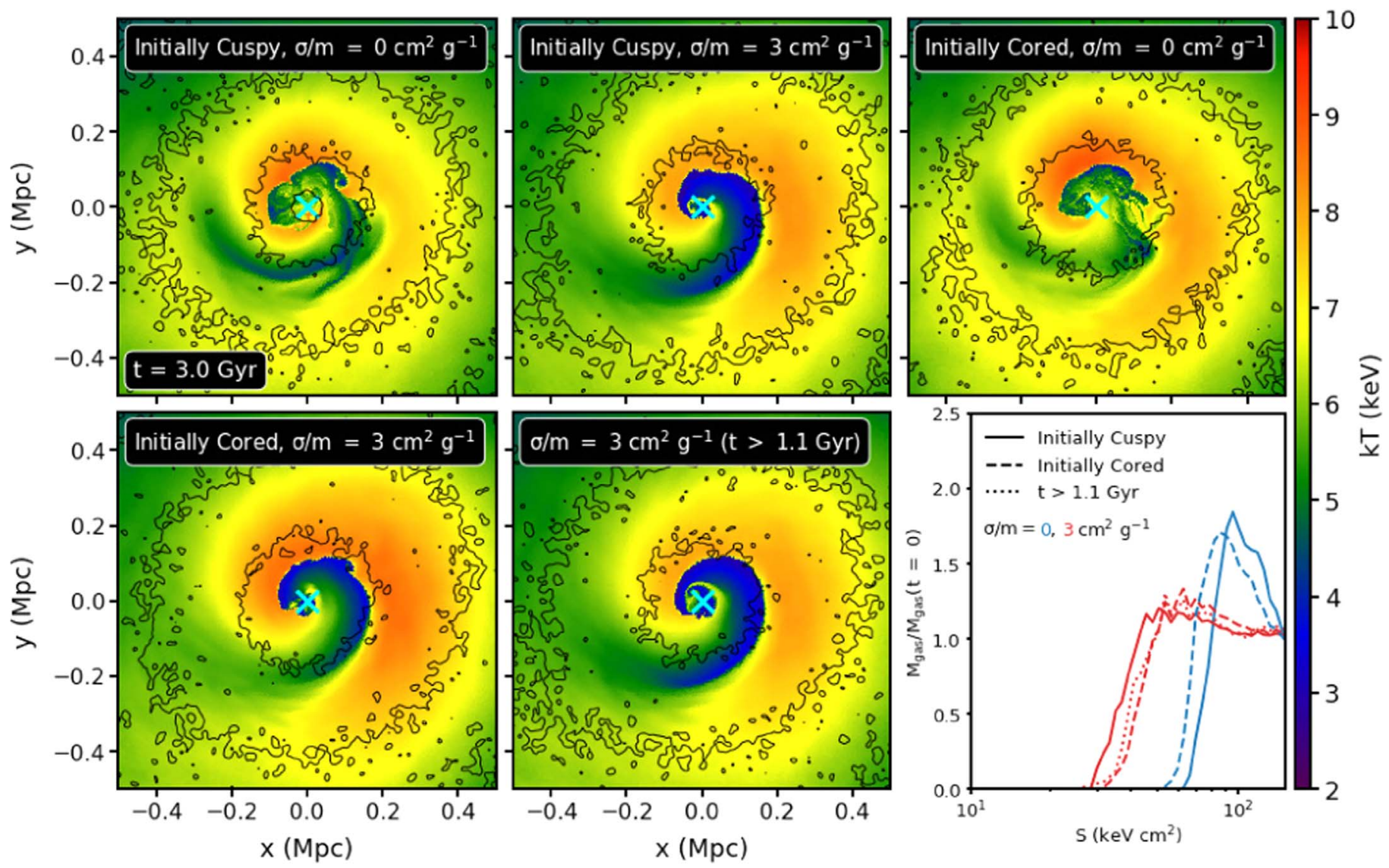

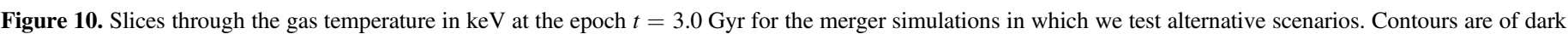

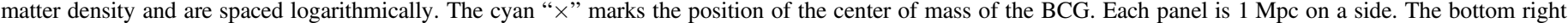
panel for each epoch shows the ratio of the mass of gas at a given entropy at that epoch to that at the same entropy at $t=0$ for the different simulations.

the initially cored simulation with $\sigma / m=0 \mathrm{~cm}^{2} \mathrm{~g}^{-1}$ (right panel). We find that in the "cuspy SIDM" simulation the enclosed mass within a $\sim 50(100) \mathrm{kpc}$ radius has been reduced by nearly $\sim 40(20)$, while in the "cored CDM" simulation the subcluster has essentially the same enclosed mass at these radii. The decrease of mass of the subcluster will both weaken the sonic wake that transfers angular momentum to the cold gas and decrease the acceleration on the main cluster core itself.

As in previous works (e.g., Ascasibar \& Markevitch 2006; ZuHone et al. 2010, 2011; Roediger et al. 2013), we used a gasless subcluster to initiate the sloshing process, which provides the cleanest setup to study the formation of cold fronts and their associated motions. To be consistent with our previous simulation investigations, the subcluster also lacks any stellar component. However, the central regions of relaxed galaxy clusters are dominated by BCGs (e.g., Newman et al. 2013a, 2013b), and the stellar component of the mass will behave collisionlessly. This concentration of mass may deepen the subcluster potential enough to reduce the stripping of DM mass from the subcluster (see, e.g., Armitage et al. 2018).

Thus, the last change to our simulation setup that we wanted to test is to include a BCG in the subcluster's core. Using Equation (2), we add a galaxy with a mass of $M_{*}=1.2 \times$ $10^{12} M_{\odot}$, which is appropriate for our subcluster mass of $2.5 \times 10^{14} M_{\odot}$ (Kravtsov et al. 2018). Our two new simulations have DM cross sections of $\sigma / m=0 \mathrm{~cm}^{2} \mathrm{~g}^{-1}$ and $\sigma / m=$ $3 \mathrm{~cm}^{2} \mathrm{~g}^{-1}$, respectively.
Figure 13 shows the appearance of the cold fronts at $t=3 \mathrm{Gyr}$ for both simulations where the subcluster has a BCG. The behavior is the same as in our previous simulations - cold fronts are more disturbed by instabilities and turbulence when there are no self-interactions. Figure 14 shows the projected DM density at the two epochs of $t=1.5$ and $3.0 \mathrm{Gyr}$ for both of these simulations. The presence of the subcluster BCG in the second simulation does not change the evolution of the subcluster's DM distribution appreciably. Thus, both of these figures show that the presence of the BCG in the subcluster does not have a significant effect on our conclusions.

This is illustrated by Figure 12. In all of the simulations in which the main cluster either begins with or develops a flat DM core, the potential is very shallow and easy for the cold, lowentropy gas to climb to larger radii (left panel of Figure 12), as was previously noted in Section 3.2.2. The cold gas climbs out to nearly the same radius by $t \sim 2.1-2.2$ Gyr. The behavior of the velocity of this cold gas is somewhat different, however. Though the increase in velocity in the simulation with $\sigma / m=0 \mathrm{~cm}^{2} \mathrm{~g}^{-1}$ and a flat DM core is delayed with respect to the same simulation with a cuspy DM core, the increase in velocity is even slower for the simulations with $\sigma / m=$ $3 \mathrm{~cm}^{2} \mathrm{~g}^{-1}$, which all exhibit similar behavior regardless of the DM core shape (right panel of Figure 12). These slower velocities result in longer growth times for KHI and noticeably smoother cold fronts. Thus, the crucial factor in the inhibition of KHI and the resulting smoothness of cold fronts and longevity of low-entropy gas in these simulations is the 

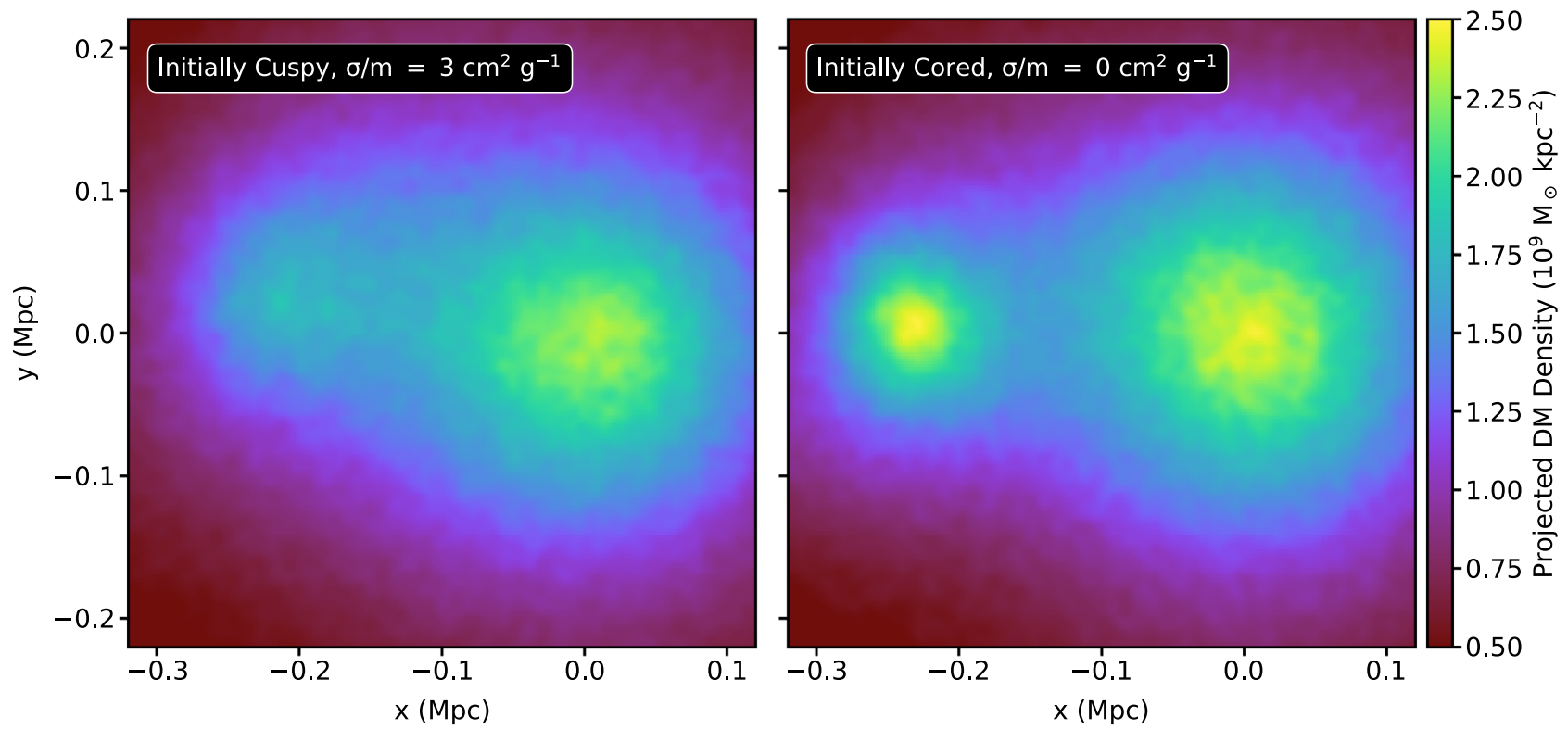

Figure 11. Projected DM density at the epoch $t=1.4 \mathrm{Gyr}$, immediately after core passage, for the initially cuspy simulation with $\sigma / \mathrm{m}^{2}=3 \mathrm{~cm}^{2} \mathrm{~g}^{-1}$ and the initially cored simulation with $\sigma / m=0 \mathrm{~cm}^{2} \mathrm{~g}^{-1}$. Each panel is $400 \mathrm{kpc}$ on a side.
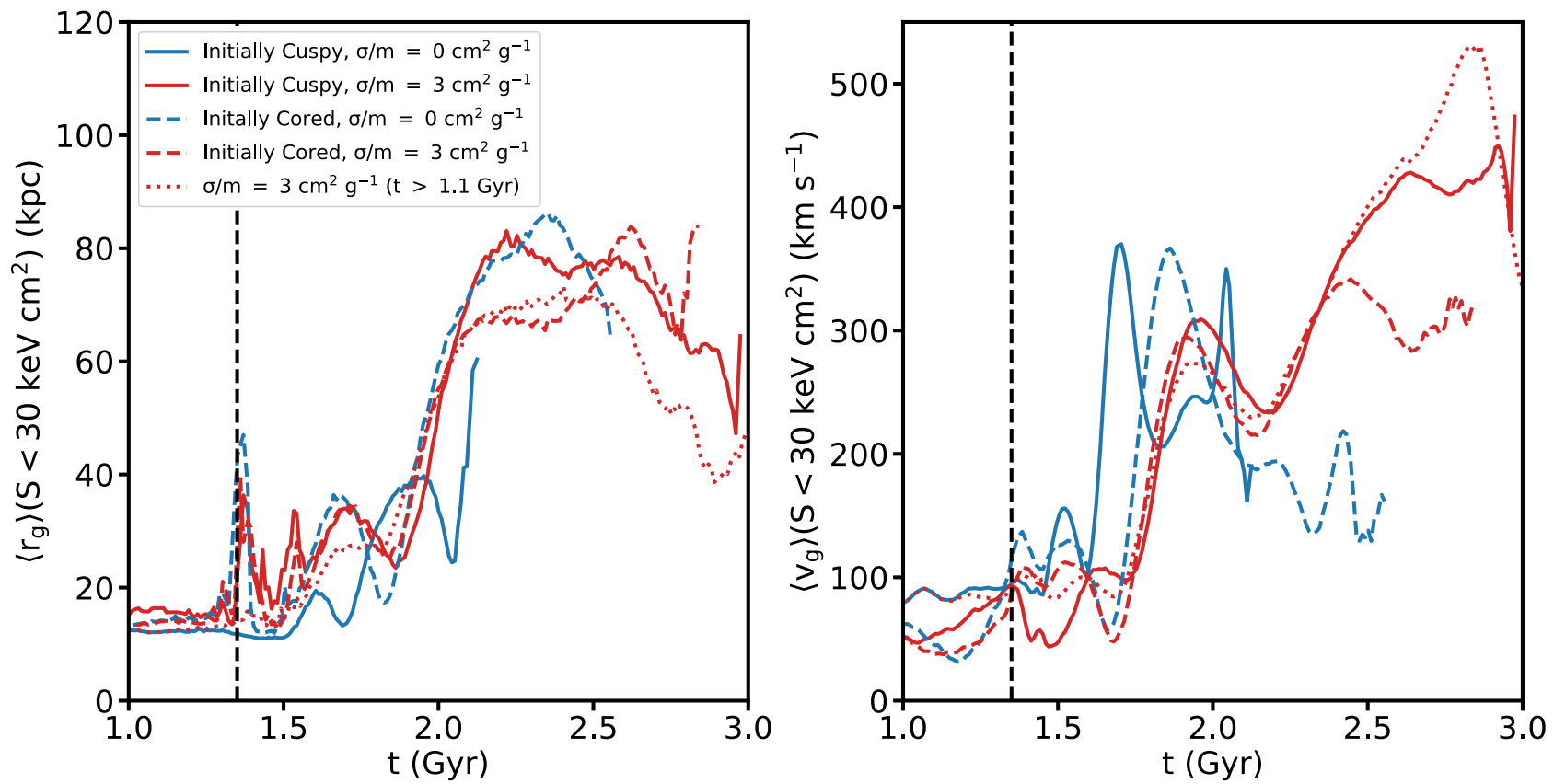

Figure 12. Evolution of the mass-weighted average radius and velocity (with respect to the center of mass frame of the BCG) of gas with $S \leqslant 30 \mathrm{keV} \mathrm{cm}^{2}$ for the simulations that test alternative scenarios. The vertical dashed line marks the approximate epoch of core passage at $t=1.35 \mathrm{Gyr}$.

decrease in the mass of the subcluster due to DM selfinteractions.

These results assumed that the DM halos of the main cluster and subcluster are in the early evolutionary stages where the cores are softening due to self-interactions. If a merger occurs during a later stage of evolution where DM cusps are developing due to gravothermal catastrophe (triggered by DM self-interactions), the results may be somewhat different. At first, it may appear that this situation would be similar to the case of collisionless DM, since both the main cluster and subcluster will have deeper gravitational potential wells.
However, it should still be expected that the subcluster will experience mass loss due to high-speed collisions of its own DM particles with those of the main cluster. This will result in a weaker influence of the subcluster on the main cluster core, and because the latter's gravitational potential well will be steeper, this will result in reduced sloshing motions.

\subsubsection{Separation between X-Ray and SZ Peaks Due to DM Self-interactions}

Because sloshing motions are subsonic, they have a minor effect on the pressure profile of the gas in cool-core clusters, 

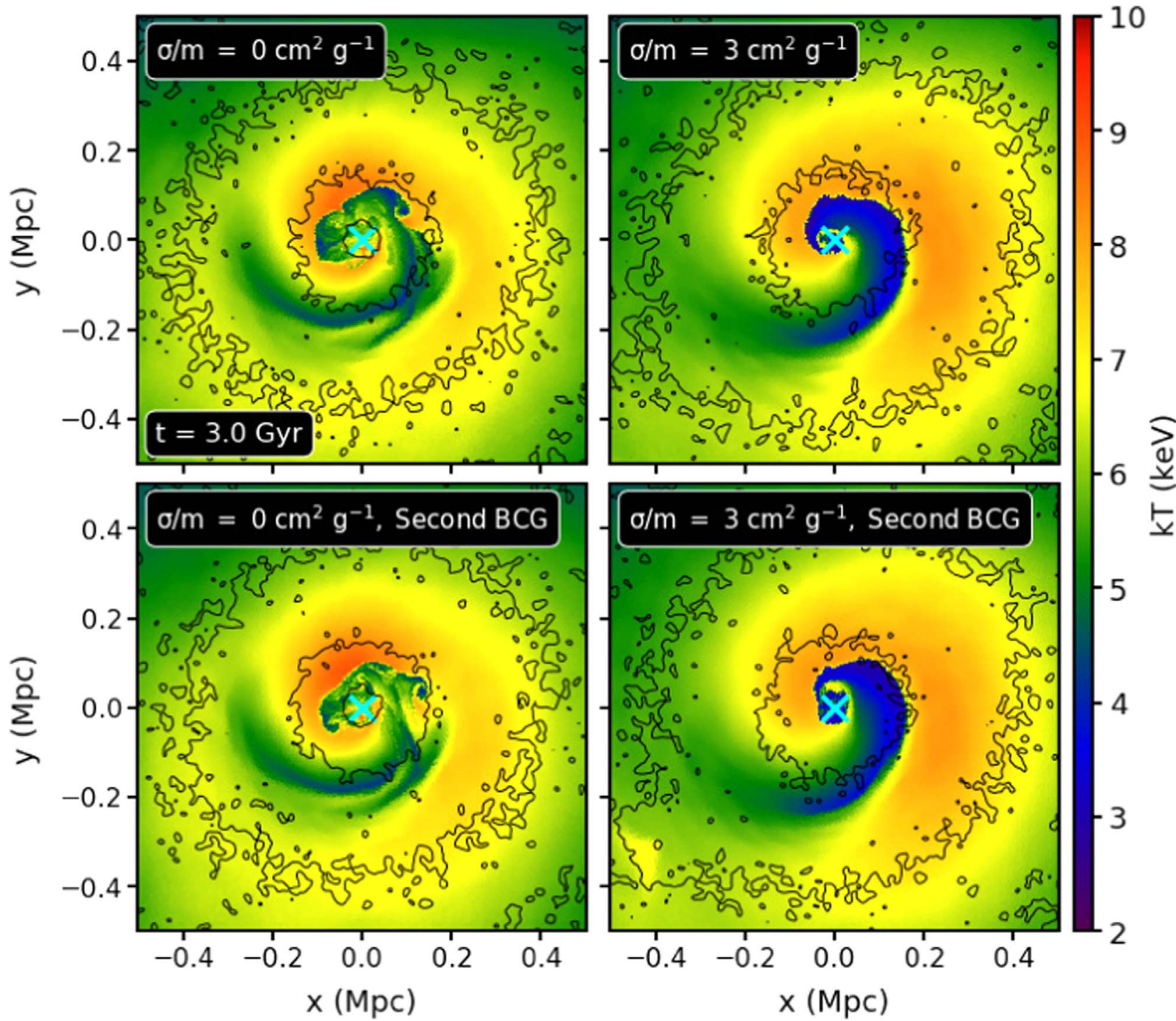

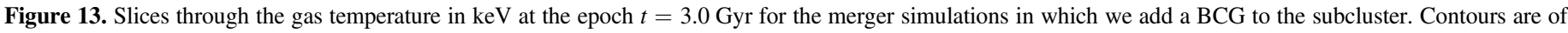

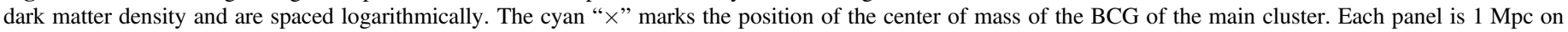
a side.

and the pressure peak remains very close to the potential minimum. The thermal Sunyaev-Zeldovich effect (hereafter $\mathrm{tSZ}$ ) is a measure of the integrated pressure along the line of sight, so the tSZ signal should be relatively unaffected by sloshing. This is seen clearly in the recent work on RXJ1347 by Ueda et al. (2018). However, the X-ray peak, which traces the densest and lowest-entropy gas in these systems, will get displaced from the cluster potential minimum. As we have already seen, this displacement reaches larger radii in simulations with non-zero DM cross section.

In Figure 15, we show maps of projected X-ray emissivity (in the $0.5-7 \mathrm{keV}$ band) with contours of the Compton tSZ parameter $y_{\mathrm{tsz}}$ overlaid for three epochs and three values of the DM cross section, which is defined by

$$
y_{\mathrm{tSZ}}=\int \frac{k_{B} T}{m_{e} c^{2}} n_{e} \sigma_{T} d \ell .
$$

The green " $x$ " in each panel marks the position of the tSZ peak. Shortly after the beginning of the sloshing process
( $t=2 \mathrm{Gyr}$ ), all of the simulations exhibit a separation between the X-ray and tSZ peak of $\sim 20-40 \mathrm{kpc}$. However, the non-zero DM cross-section simulations allow for greater and more longlived separations between the tSZ and X-ray peaks. Without self-interactions, the two peaks already again coincide by $t=3 \mathrm{Gyr}$, but in the most extreme case shown of $\sigma / m=$ $3 \mathrm{~cm}^{2} \mathrm{~g}^{-1}$, a separation between the two peaks of $\sim 80 \mathrm{kpc}$ persists even to $t=4 \mathrm{Gyr}$. This implies that for a given cluster a separation between these two peaks may provide an independent way to constrain the value of $\sigma / m$. This, however, would require knowledge of the stage of the sloshing motions, and would require a numerical simulation dedicated to matching the conditions of a particular cluster. Alternatively, this question could be addressed by running a large number of simulations over a wide parameter space in self-interaction cross section, mass ratio, and impact parameter, which would place tighter constraints on such X-ray/SZ separations due to SIDM. 

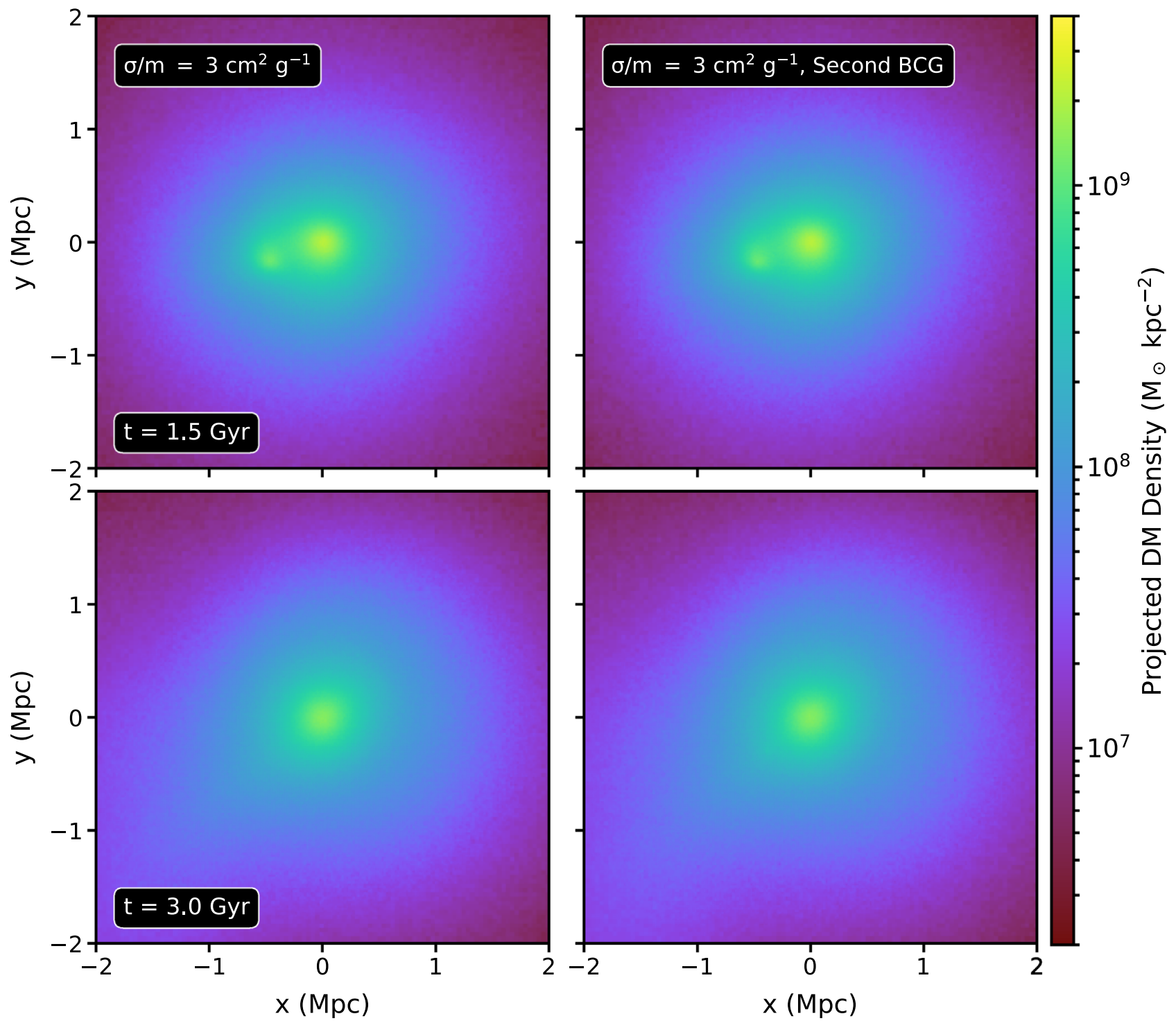

Figure 14. Projected DM density at the epochs $t=1.5,3.0$, and $4.0 \mathrm{Gyr}$ for two simulations with $\sigma / m=3 \mathrm{~cm}^{2} \mathrm{~g}^{-1}$, where the subcluster is with (right panel) or without (left panel) a BCG. Each panel is $4 \mathrm{Mpc}$ on a side.

\section{Summary}

We have performed a suite of simulations of core gas sloshing in a galaxy cluster core, building on previous work by adding the effect of DM self-interactions. The key ingredient in forming sloshing cold fronts in cluster cores via interactions with smaller clusters is the radically different collisionalities of the DM and baryonic components. Thus, the effect of a small DM cross section could potentially produce observable consequences on the formation and evolution of these features. Our main results are as follows:

1. In agreement with previous works, isolated cool-core clusters with initially cuspy DM density profiles gradually evolve flatter DM cores via DM self-interactions. The resulting gradual change in the gravitational potential causes a slow, adiabatic expansion and cooling of the gas in the center of the cluster. These changes are modest, so the essential thermodynamic structure of the cluster remains intact.

2. Sloshing cold fronts form in the same manner when the DM cross-section is non-zero as in the collisionless case. Due to the adiabatic cooling of the gas from the softening of the core, the sloshing gas is colder in simulations with larger DM cross-section. The cold fronts in simulations with significant self-interactions are also less susceptible to the effects of KHI and turbulent mixing, at least in the earlier stages.

3. In the earliest stages, the flattening of the potential caused by self-interactions enables the lowest-entropy gas to reach larger radii because there is a shallower potential to climb. On the other hand, because of the frequent and high-speed collisions the DM within subcluster experiences upon infall, it experiences further flattening of its potential and mass loss, and thus its influence on the core gas is weakened. The result is that the speed of the sloshing motions is therefore slower in the presence of self-interactions, which explains the slower growth of KHI in these simulations. The slower growth of turbulence and instabilities results in less turbulent mixing within the core region, and hence the lowest-entropy gas is longer-lasting.

4. Large impact-parameter encounters with small subclusters do not produce a significant additional flattening of the larger cluster's core DM profile beyond what already 

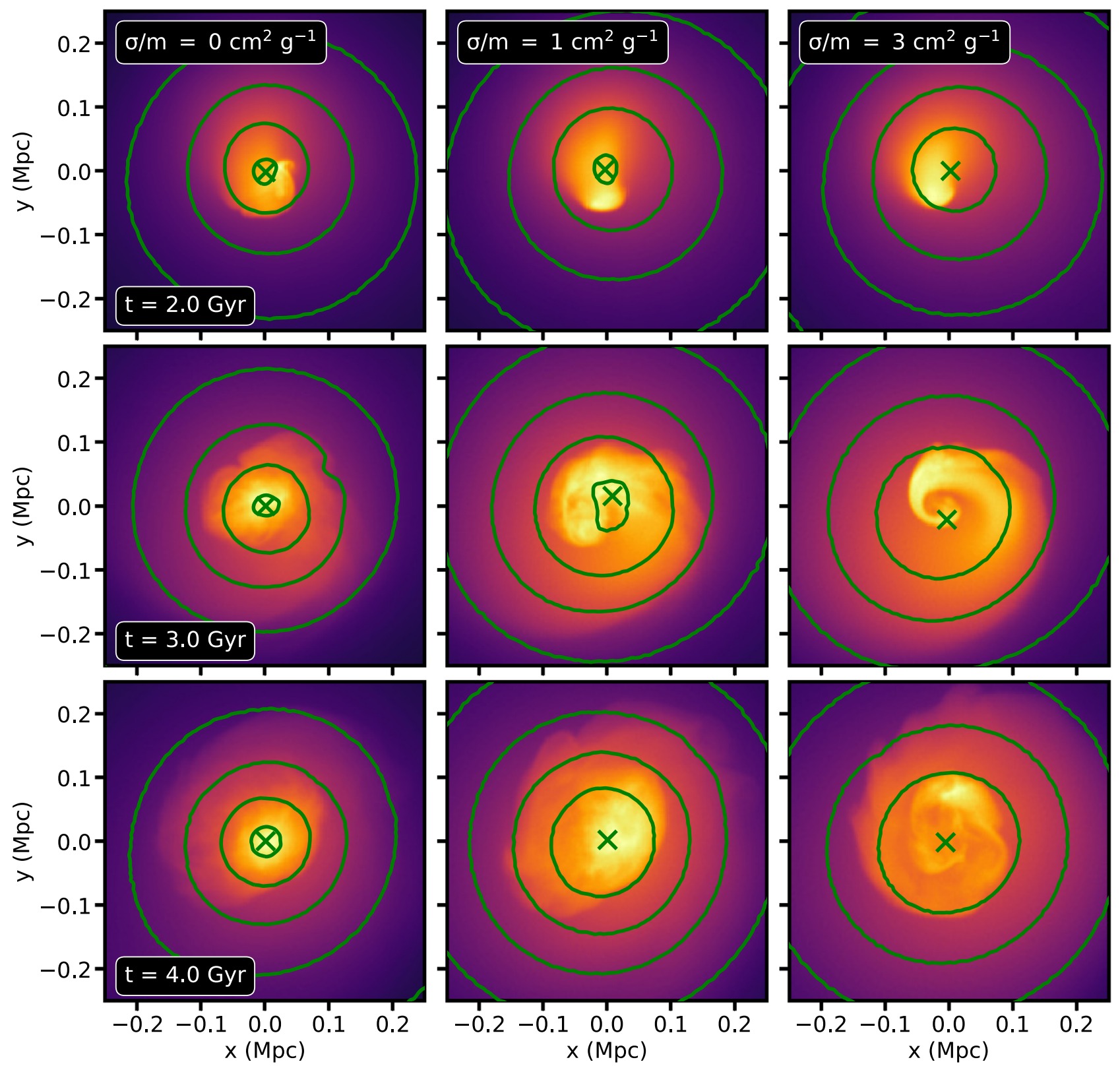

Figure 15. Projected X-ray emissivity in the $0.5-7 \mathrm{keV}$ band (in arbitrary units) with $y_{\mathrm{tSz}}$ contours overlaid for three different values of $\sigma / m$ at the epochs $t=2,3$, and 4 Gyr. The green " $\times$ " symbol marks the position of the tSZ peak. Each panel is $0.5 \mathrm{Mpc}$ on a side. The color scales for the X-ray emission are different in each panel and are chosen to clearly show the morphology.

has occurred due to its own self-interactions. However, interactions with the larger cluster's DM particles strip the subcluster of its DM and mix it in with the main cluster's DM on shorter timescales than would otherwise occur due to dynamical friction alone.

5. The flattening of the DM core by self-interactions can result in significant separations between X-ray and SZ peaks which can persist for a number of Gyr.

These conclusions lead to the obvious question-may they be used to constrain the value of $\sigma / m$ on cluster scales? The slower growth of KHI in the presence of DM self-interactions compared to the same merger scenario as collisionless DM is an intriguing result, especially considering most sloshing cold fronts appear to be relatively smooth. The reduced presence of KHI in observations with respect to their ubiquity in hydrodynamic simulations of cold fronts has typically been explained by magnetic fields (ZuHone et al. 2011) or viscosity (Roediger et al. 2013). Magnetic fields are observed in clusters, and the viscosity of the ICM is still unknown, so there are far too many uncertainties to make any definite conclusions in this regard. Because the flattening of the inner density profile allows for the cold gas to climb to higher radii, a large sample of clusters could be examined for trends in the central density slope versus the radial extent of the sloshing gas. As mentioned above, SIDM may provide a partial explanation for the difficulty in easily locating subclusters that initiate the sloshing process, but this would require a more extensive study of the galaxy and lensing maps around many clusters with sloshing cold fronts.

Perhaps the most stringent constraints on the SIDM crosssection could be placed by investigating separations between $\mathrm{X}$-ray and SZ peaks in a sample of relaxed clusters with sloshing cold fronts, using high-angular-resolution SZ experiments in 
conjunction with X-ray observations. Such large peak separations should be correlated with the presence of shallower DM cores that have been softened by DM self-interactions, which may be discerned by lensing analyses. For larger SIDM cross sections, the peak separation lasts longer, which implies that peak separations will be seen in conjunction with cold fronts at larger radii. Significant peak separations should be on the order of $\sim 1$ gas core radii for a given cluster ( $60 \mathrm{kpc}$ in our case), as suggested by Figure 15. If the self-interaction cross section increases with smaller characteristic velocities and thus less massive halos, then it may be expected that such peak separations would be more prevalent for smaller clusters and large groups. Such effects should also be looked for in cosmological simulations including DM self-interactions (Robertson et al. 2018, 2019).

Our idealized setup comes with a number of limitations that must be noted. Our initial conditions, which begin with two single NFW-like cuspy DM halos, are inherently out of equilibrium in the presence of self-interactions. This was evidenced by their transition to cored DM profiles in the run-up to the first core passage of the merger, and more or less remained in that state for the duration of the evolution of the cold fronts. This transition also resulted in adiabatic cooling and expansion of the gas, which had an effect on the temperature of the cold fronts, which was different in the different simulations. In a more realistic cosmological context, clusters are undergoing continuous accretion and merging with other clusters and groups, and the clusters themselves contain numerous smaller substructures down to galaxy scales. In this setting, the properties of the DM cores of clusters are likely to be more dynamic, becoming more core-like by self-interactions and more cusp-like via gravothermal collapse. Also, we have not included additional gas physics such as cooling, feedback, and star formation in these simulations. In these circumstances, gas would cool, condense, and form stars in the cluster center, which would have the effect of deepening the potential well and rendering the mass profile more cusp-like. A fully self-consistent picture would include all of these effects together, as in Robertson et al. (2018, 2019). Studying gas motions in the core of a cluster with DM self-interactions in such a cosmological context is left for future work.

It should also be noted that on longer timescales than those considered in this work SIDM halos eventually undergo a gravothermal catastrophe and recollapse, which would produce a cusp-like DM density profile in the central region (Kochanek \& White 2000). This process will be sped up for inelastic collisions (Essig et al. 2018). Under such conditions, the potential would evolve during the cluster merger on a faster timescale and the effects on the ICM are likely to be more complex.

Future papers will investigate the effects of DM selfinteractions on the X-ray emitting plasma in major as well as minor mergers, examining both the thermodynamic and kinematic properties of the latter during the merger.

This work required the use and integration of a number of Python software packages for science, including AstroPy (Astropy Collaboration et al. 2013), ${ }^{5}$ Matplotlib (Hunter 2007), ${ }^{6}$ NumPy, ${ }^{7}$ SciPy, ${ }^{8}$ and yt (Turk et al. 2011). ${ }^{9}$ We are thankful to

\footnotetext{
5 http://www.astropy.org

6 http://matplotlib.org

7 http://www.numpy.org

8 http://www.scipy.org/scipylib/

9 http://yt-project.org
}

the developers of these packages. We also thank Volker Springel for the use of the AREPO code. J.A.Z. thanks David Barnes, Rahul Kannan, Federico Marinacci, and Hui Li for useful discussions. He also thanks Grant Tremblay for assistance with a last-minute fix to a figure. J.A.Z. acknowledges support through Chandra Award Number G04-15088X issued by the Chandra X-ray Center, which is operated by the Smithsonian Astrophysical Observatory for and on behalf of NASA under contract NAS8-03060. J.Z. acknowledges support by a Grant of Excellence from the Icelandic Research Fund (grant No. 173929-051). The numerical simulations were performed using the computational resources of the Advanced Supercomputing Division at NASA/Ames Research Center.

\section{ORCID iDs}

\section{J. A. ZuHone (iD https://orcid.org/0000-0003-3175-2347}

\section{References}

Andrade, K. E., Minor, Q., Nierenberg, A., \& Kaplinghat, M. 2019, MNRAS, 487, 1905

Armitage, T. J., Barnes, D. J., Kay, S. T., et al. 2018, MNRAS, 474, 3746

Ascasibar, Y., \& Markevitch, M. 2006, ApJ, 650, 102

Astropy Collaboration, Robitaille, T. P., Tollerud, E. J., et al. 2013, A\&A, 558, A33

Benson, A. J., Lacey, C. G., Baugh, C. M., Cole, S., \& Frenk, C. S. 2002 , MNRAS, 333, 156

Bovill, M. S., \& Ricotti, M. 2009, ApJ, 693, 1859

Boylan-Kolchin, M., Bullock, J. S., \& Kaplinghat, M. 2011, MNRAS, 415, L40

Boylan-Kolchin, M., Bullock, J. S., \& Kaplinghat, M. 2012, MNRAS, 422, 1203

Bullock, J. S., \& Boylan-Kolchin, M. 2017, ARA\&A, 55, 343

Bullock, J. S., Kravtsov, A. V., \& Weinberg, D. H. 2000, ApJ, 539, 517

Chen, H., Jones, C., Andrade-Santos, F., ZuHone, J. A., \& Li, Z. 2017, ApJ, 838,38

Clarke, T. E., Blanton, E. L., \& Sarazin, C. L. 2004, ApJ, 616, 178

Cyr-Racine, F.-Y., Sigurdson, K., Zavala, J., et al. 2016, PhRvD, 93, 123527

Davé, R., Thompson, R., \& Hopkins, P. F. 2016, MNRAS, 462, 3265

Despali, G., Sparre, M., Vegetti, S., et al. 2019, MNRAS, 484, 4563

Díaz Rivero, A., Dvorkin, C., Cyr-Racine, F.-Y., Zavala, J., \& Vogelsberger, M. 2018, PhRvD, 98, 103517

Dubois, Y., Pichon, C., Welker, C., et al. 2014, MNRAS, 444, 1453

Eddington, A. S. 1916, MNRAS, 76, 572

Efstathiou, G. 1992, MNRAS, 256, 43P

Essig, R., McDermott, S. D., Yu, H.-B., \& Zhong, Y.-M. 2018, arXiv:1809. 01144

Flores, R. A., \& Primack, J. R. 1994, ApJL, 427, L1

Ghizzardi, S., Rossetti, M., \& Molendi, S. 2010, A\&A, 516, A32

Gnedin, O. Y., \& Ostriker, J. P. 2001, ApJ, 561, 61

Harvey, D., Robertson, A., Massey, R., \& McCarthy, I. G. 2019, MNRAS, 488, 1572

Hernquist, L. 1990, ApJ, 356, 359

Hunter, J. D. 2007, CSE, 9, 90

Johnson, R. E., Markevitch, M., Wegner, G. A., Jones, C., \& Forman, W. R. 2010, ApJ, 710, 1776

Kahlhoefer, F., Schmidt-Hoberg, K., Frandsen, M. T., \& Sarkar, S. 2014, MNRAS, 437, 2865

Kaplinghat, M., Tulin, S., \& Yu, H.-B. 2016, PhRvL, 116, 041302

Kazantzidis, S., Magorrian, J., \& Moore, B. 2004, ApJ, 601, 37

Kim, S. Y., Peter, A. H. G., \& Wittman, D. 2017, MNRAS, 469, 1414

Kochanek, C. S., \& White, M. 2000, ApJ, 543, 514

Koda, J., \& Shapiro, P. R. 2011, MNRAS, 415, 1125

Kravtsov, A. V., Vikhlinin, A. A., \& Meshcheryakov, A. V. 2018, AstL, 44, 8

Kuzio de Naray, R., Martinez, G. D., Bullock, J. S., \& Kaplinghat, M. 2010, ApJL, 710, L161

Lovell, M. R., Zavala, J., Vogelsberger, M., et al. 2018, MNRAS, 477, 2886

Lynden-Bell, D., \& Wood, R. 1968, MNRAS, 138, 495

Madau, P., Shen, S., \& Governato, F. 2014, ApJL, 789, L17

Markevitch, M., Gonzalez, A. H., Clowe, D., et al. 2004, ApJ, 606, 819

Markevitch, M., Gonzalez, A. H., David, L., et al. 2002, ApJL, 567, L27

Markevitch, M., Ponman, T. J., Nulsen, P. E. J., et al. 2000, ApJ, 541, 542 
Markevitch, M., \& Vikhlinin, A. 2007, PhR, 443, 1

Markevitch, M., Vikhlinin, A., \& Forman, W. R. 2003, in ASP Conf. Ser. 301, Matter and Energy in Clusters of Galaxies, ed. S. Bowyer \& H. Chorng-Yuan (San Francisco, CA: ASP), 37

Mashchenko, S., Wadsley, J., \& Couchman, H. M. P. 2008, Sci, 319, 174

McCarthy, I. G., Bird, S., Schaye, J., et al. 2018, MNRAS, 476, 2999

Meneghetti, M., Yoshida, N., Bartelmann, M., et al. 2001, MNRAS, 325, 435

Merritt, D., Graham, A. W., Moore, B., et al. 2006, AJ, 132, 2685

Moore, B. 1994, Natur, 370, 629

Moore, B., Quinn, T., Governato, F., Stadel, J., \& Lake, G. 1999, MNRAS, 310,1147

Navarro, J. F., Frenk, C. S., \& White, S. D. M. 1997, ApJ, 490, 493

Newman, A. B., Treu, T., Ellis, R. S., et al. 2013a, ApJ, 765, 24

Newman, A. B., Treu, T., Ellis, R. S., \& Sand, D. J. 2013b, ApJ, 765, 25

Oman, K. A., Navarro, J. F., Fattahi, A., et al. 2015, MNRAS, 452, 3650

Oñorbe, J., Boylan-Kolchin, M., Bullock, J. S., et al. 2015, MNRAS, 454, 2092

Peter, A. H. G., Rocha, M., Bullock, J. S., \& Kaplinghat, M. 2013, MNRAS, 430, 105

Pontzen, A., \& Governato, F. 2012, MNRAS, 421, 3464

Power, C., Navarro, J. F., Jenkins, A., et al. 2003, MNRAS, 338, 14

Randall, S. W., Markevitch, M., Clowe, D., et al. 2008, ApJ, 679, 1173

Read, J. I., Agertz, O., \& Collins, M. L. M. 2016, MNRAS, 459, 2573

Read, J. I., Walker, M. G., \& Steger, P. 2019, MNRAS, 484, 1401

Robertson, A., Harvey, D., Massey, R., et al. 2019, MNRAS, 488, 3646

Robertson, A., Massey, R., \& Eke, V. 2017a, MNRAS, 465, 569

Robertson, A., Massey, R., \& Eke, V. 2017b, MNRAS, 467, 4719

Robertson, A., Massey, R., Eke, V., et al. 2018, MNRAS, 476, L20

Roediger, E., Brüggen, M., Simionescu, A., et al. 2011, MNRAS, 413, 2057

Roediger, E., Kraft, R. P., Forman, W. R., et al. 2013, ApJ, 764, 60
Roediger, E., Lovisari, L., Dupke, R., et al. 2012, MNRAS, 420, 3632 Sawala, T., Frenk, C. S., Fattahi, A., et al. 2016, MNRAS, 457, 1931

Schaye, J., Crain, R. A., Bower, R. G., et al. 2015, MNRAS, 446, 521

Schaye, J., Dalla Vecchia, C., Booth, C. M., et al. 2010, MNRAS, 402, 1536

Simionescu, A., Werner, N., Forman, W. R., et al. 2010, MNRAS, 405, 91

Spergel, D. N., \& Steinhardt, P. J. 2000, PhRvL, 84, 3760

Springel, V. 2010, MNRAS, 401, 791

Springel, V., Pakmor, R., Pillepich, A., et al. 2018, MNRAS, 475, 676

Springel, V., White, S. D. M., Jenkins, A., et al. 2005, Natur, 435, 629

Todoroki, K., \& Medvedev, M. V. 2019a, MNRAS, 783, 3983

Todoroki, K., \& Medvedev, M. V. 2019b, MNRAS, 483, 4004

Tulin, S., \& Yu, H.-B. 2018, PhR, 730, 1

Turk, M. J., Smith, B. D., Oishi, J. S., et al. 2011, ApJS, 192, 9

Ueda, S., Kitayama, T., Oguri, M., et al. 2018, ApJ, 866, 48

Vikhlinin, A., Markevitch, M., Murray, S. S., et al. 2005, ApJ, 628, 655

Vogelsberger, M., Genel, S., Springel, V., et al. 2014a, Natur, 509, 177

Vogelsberger, M., Genel, S., Springel, V., et al. 2014b, MNRAS, 444, 1518

Vogelsberger, M., \& Zavala, J. 2013, MNRAS, 430, 1722

Vogelsberger, M., Zavala, J., Cyr-Racine, F.-Y., et al. 2016, MNRAS, 460, 1399

Vogelsberger, M., Zavala, J., \& Loeb, A. 2012, MNRAS, 423, 3740

Vogelsberger, M., Zavala, J., Schutz, K., \& Slatyer, T. R. 2019, MNRAS, 484, 5437

Vogelsberger, M., Zavala, J., Simpson, C., \& Jenkins, A. 2014c, MNRAS, 444, 3684

Zavala, J., Vogelsberger, M., \& Walker, M. G. 2013, MNRAS, 431, L20

ZuHone, J. A., Markevitch, M., \& Johnson, R. E. 2010, ApJ, 717, 908

ZuHone, J. A., Markevitch, M., \& Lee, D. 2011, ApJ, 743, 16

ZuHone, J. A., \& Roediger, E. 2016, JPIPh, 82, 53582030 